\title{
Kompetenzstreben und Kompetenzerwerb: Funktionale didaktische Fördermöglichkeiten durch Differenzierung und Individualisierung
}

\author{
HANS-JÖRG HERBER UND ÉVA VÁSÁRHELYI
}

Abstract. As a first glimpse of specific research endeavours the most important components of competence motivation are discussed in relation to didactical questions of gaining competence by inner differentiation and individualization: self-efficacy, optimal challenge, intrinsic motivation, exploration needs, internal attribution, self-determination motivation, defense of self-worth, self-concept, and achievement motivation. In this sense "competence" means ever changing standards of self-regulation of an individual interacting with the various cognitive and emotional demands of his/her environment.

In fulfilling these requirements a prototypical example of inner differentiation in mathematics instruction is given. This didactical elaboration is available as a selfinstructing unit in Hungarian and German language within the "Electronic periodical of the Department of Methodology of Mathematics" which can be reached under http://mathdid.inhun.com.

Key words and phrases: psychology of learning and motivation, competence, computer based problem solving, worksheets, visualizations.

ZDM Subject Classification: C20, C30, C40, D50, U50, U60.

Diese Arbeit fußt auf gemeinsamen Vorträgen der Autoren im Rahmen des österreichischungarischen Dissertantenseminars in den Jahren 2003-2005. Ein Vorabdruck dieses Artikels erschien - in leicht modifizierter Form - in den Salzburger Beiträgen zur Erziehungswissenschaft (Herber \& Vásárhelyi 2004).

Copyright (C) 2006 by University of Debrecen 


$$
\text { "herber" — 2006/6/26 - 10:27 — page } 2-\# 2
$$

1. Kognitive, emotionale und motivationale Determinanten von Kompetenzstreben und Kompetenzerwerb

Neuropsychologisch können drei Ebenen des Kompetenzstrebens und Kompetenzerwerbs unterschieden werden:

a) eine evolutionstheoretisch begründete Anpassungsstrategie (genetische Determination) an spezifische Lebensbedingungen („survival of the fittest“, vgl. Riedl 1980, Lumsden \& Gushurst 1985, Klix 1992, 1993, Kimble 1993, Slusarek 1995, Schurz 2001, Wiesner 2004);

b) frühe Lebenserfahrungen, die das neuronale Netzwerk formen („Was gebraucht wird, entwickelt sich und will weiterhin gebraucht werden." Vgl. z.B. Freud 1895/1962, Greenfield 1999, Singer 2002);

c) Lernen im Erwachsenenalter: Emotionale und kognitive Herausforderungen motivieren, welche Netzwerkverbindungen gefestigt und ausgebaut oder geschwächt („,vergessen“) werden (Greenfield 1999, Pöppel 2000, Edelmann \& Tononi 2002, Damasio 2003).

Moderne psychologische und pädagogische Forschung nimmt mehr und mehr Bezug auf neurophysiologische Forschungsergebnisse, kontrolliert damit die genuin psychologischen Systembildungen und diese befruchten umgekehrt neuropsychologische Forschungsbemühungen (z.B. Kuhl 2001, Standop 2002).

\subsection{Kompetenzstreben}

White (1959) führte das Kompetenzstreben als globale intrinsische Motivation ein, mit der Umwelt effektiv umzugehen. Es ist nicht an spezifische Triebe oder Bedürfnisse gebunden und somit im Sinne von Lewin (1926) ein „Quasibedürfnis" des gesamten Organismus, das nicht durch die Befriedigung einzelner Triebe, Bedürfnisse, Motive, Interessen, etc. im Sinne von Hull (1943) oder Freud (1904-5/1965) gestillt werden kann: „... competence will refer to an organism's capacity to interact effectively with its environment ... motivation needed to attain competence cannot be wholly derived from sources of energy currently conceptualized as drives or instincts." (White 1959, 297) Das Kompetenzstreben kann sich auf ganz unterschiedliche Verhaltensweisen beziehen (z.B. Objekte visuell fixieren bei Babies, Türme aus Bauklötzen bauen bei Zweijährigen, mit dem Computer spielen bei Zehnjährigen, einen wissenschaftlichen Artikel schreiben bei Erwachsenen, etc.). 
Whites Kompetenzmotivation resultiert aus einem evolutionären Anpassungsvorteil, weil es den Organismus „antreibt“, immer effektiver mit sich selbst und der Umwelt umzugehen. Mindestens beim Menschen ist dazu lebenslanges Lernen erforderlich.

\subsubsection{Selbstwirksamkeit}

Als eine kognitionspsychologische Interpretation der Kompetenzmotivation sensu White (1959) kann die Theorie der Selbstwirksamkeit (self-efficacy) von Bandura (1997) aufgefasst werden. Whites Kompetenzstreben bezieht sich im Grunde auf alle lebensrelevanten Situationen von Tieren und Menschen, die Untersuchungen des Bandura-Kreises beziehen sich vor allem auf schulische, insbesondere intellektuelle Leistungen. Typischer Weise heben einschlägige (domainspezifische) Erfolge die entsprechende Selbstwirksamkeitsüberzeugung an, Misserfolgserfahrungen senken sie. Doch sind Selbstwirksamkeitsüberzeugungen - wie andere Motivationen auch (vgl. Herber 1998a, 2004, Kuhl 2001, Schmuck \& Sheldon 2001, Herber \& Vásárhelyi 2002a, 2003, Leary \& Tangney 2003, StiensmeierPelster \& Rheinberg 2003, Rheinberg 2004, Dresel 2004) - keine 1:1-Abbildungen bisheriger (objektiver) Erfahrungen. Selbstwirksamkeitsüberzeugungen entstehen - angestoßen durch aktuelle und erinnerte Erfahrungen - durch hochinferentes (abstrahierendes) Denken und Fühlen. Diese kognitiv-emotionalen Prozesse werden beeinflusst durch frühere Überzeugungen, vergangene und gegenwärtige Erwartungen, wahrgenommene Aufgabenschwierigkeiten, Anstrengungsaufwand, erhaltene Hilfeleistung durch andere, etc. (Erfolg führt z.B. nicht zu erhöhter Selbstwirksamkeit, wenn die Aufgabe als sehr leicht oder der Anstrengungsaufwand als übermäßig hoch empfunden wurde, vgl. Dresel 2004). Auch erhaltene Hilfeleistung durch andere stärkt nicht gerade die Selbstwirksamkeitsüberzeugung. Andererseits kann durch Modelllernen bzw. stellvertretende Bekräftigung (Bandura 1976) die Selbstwirksamkeitsüberzeugung gestärkt werden (wenn eine „gleichgeartete" Person etwas schafft, worin man wenig eigene Erfahrungen hat und daher unsicher ist). Auch überzeugende Reattribuierungen („Du schaffst es schon, wenn Du nur willst!“) durch Personen, denen man vertraut und die man in solchen Urteilen für kompetent hält, kann den Glauben an die eigene Selbstwirksamkeit erhöhen (Dresel 2004). Angstbedingte Übererregung hingegen senkt die Selbstwirksamkeitsüberzeugung und damit die Wahrscheinlichkeit, eine Aufgabe erfolgreich lösen zu können (z.B. Meyer 2000). 


$$
\text { "herber" — 2006/6/26 - 10:27 — page } 4 \text { - \#4 }
$$

In diesem Sinne wahrgenommene Selbstwirksamkeit kann Kognitionen und Emotionen in Leistungssituationen wesentlich beeinflussen. Schüler (jedweden Alters), die sich nicht kompetent genug fühlen eine bestimmte Aufgabe zu lösen, geraten leicht in Versagensangst und entwickeln Zwangsgedanken zu versagen - besonders in Bewertungssituationen. Ihre Energie wird in ängstlicher Selbstaufmerksamkeit gebunden, während Kommilitonen mit hoher Selbstwirksamkeitsüberzeugung aufgabenorientiert immer bessere Problemlösestrategien entwickeln (vgl. analoge Befunde in der Leistungsmotivationsforschung, z.B. Nicholls et al. 1989, Kuhl 2001, Herber \& Vásárhelyi 2003). Hohe Selbstwirksamkeitsüberzeugung (verbunden mit Selbstkompatibilität) fördert die Sensitivität für Probleme, eine flexible Entwicklung von Problemlösestrategien und selbstgesteuertes Lernen (Pintrich \& De Groot 1990, Leutner et al. 2001, Kuhl 2001, Kazén et al. 2003, Fröhlich \& Kuhl 2003).

Auch die Selbstwirksamkeitsüberzeugungen von Lehrern haben Einfluss auf die Entwicklung des Kompetenzstrebens von Schülern. Lehrer mit hoher Selbstwirksamkeitsüberzeugung sind proaktiv und sehen mehr Sinn in ihrer Tätigkeit, „,brennen weniger leicht aus“, kontrollieren weniger das Verhalten der Schüler und führen diese zu mehr Erfolg, sind geduldiger bei Lernschwierigkeiten und analysieren selbstkritischer ihre Lehrmethoden (Ashton 1985, Ashton \& Webb 1986, Schmitz \& Schwarzer 2000, Dresel 2004).

\subsubsection{Optimale Herausforderung}

Kompetenzmotivation entsteht nur, wenn Aufgaben eine gewisse („mittlere“) Herausforderung haben, an deren Bewältigung man wachsen und seine Kompetenzen weiter entwickeln kann (Atkinson 1957, Herber 1976, 1979, 1998a, Eysenck 1982, Thayer 1987, 1989, Strelau \& Eysenck 1987, Kuhl 2001, Schimmack \& Reisenzein 2002, Herber \& Vásárhelyi 2003, etc.). Die Kompetenzmotivation „springt nicht an", wenn Routinetätigkeiten oder Aufgaben zu machen sind, die man bereits gut kann und die daher als leicht eingeschätzt werden. Ein Kleinkind dreht immer wieder an einem Türknopf, bis es durch diese Tätigkeit die Tür sicher öffnen kann, dann verliert es das Interesse daran. Wenn Puzzles immer ein wenig schwerer werden als das zuletzt erfolgreich bewältigte Puzzle, dann arbeiten Kinder länger daran, als wenn der Schwierigkeitsgrad der auf einander folgenden Puzzles immer gleich bleibt (McMullin \& Steffen 1982; für schulische Aufgaben gilt das gleiche Prinzip, vgl. Herber \& Vásárhelyi 2002b, 2003). Bei den Spielen der Erwachsenen macht es in analoger Weise wenig Spaß, z.B. jemanden beim Tennis zu schlagen, der wesentlich schwächer spielt. 


$$
\text { "herber" — 2006/6/26 - 10:27 — page } 5-\# 5
$$

$\underline{\text { Kompetenzstreben und Kompetenzerwerb: Funktionale didaktische Fördermöglichkeiten. . . } 5}$

\subsubsection{Intrinsische Motivation}

Erfolg bei (optimal) herausfordernden Tätigkeiten stärkt nicht nur das Kompetenzstreben und Gefühle der Selbstwirksamkeit, sondern auch die intrinsische Motivation, wobei extrinsische Motivation, also nicht sachbezogene Verstärkungen (Geldzuwendungen, soziale Anerkennung, Prestige- bzw. Machtgewinn) die intrinsische Motivation korrumpieren können (siehe dazu theoretische Argumente und empirische Befunde in z.T. unterschiedlichen Systemzusammenhängen, z.B. Deci 1975, 1996, Herber 1977, 1979, 1998a, Herber et al. 1999, Deci et al. 2001, Kasser \& Ryan 2001, Kuhl 2001, Vansteenkiste \& Deci 2003, Fox \& Calkins 2003, Rheinberg 2004).

Wer sich - z.B. bei schulischen Aufgabenstellungen - kompetent fühlt, ist in diesem Bereich mehr intrinsisch motiviert als Kollegen mit weniger Kompetenzund Selbstwirksamkeitsgefühlen. Tätigkeitsbereiche, in denen das Kompetenzund Selbstwirksamkeitsstreben befriedigt wird und die Anforderungen intrinsisch motivierend erlebt werden, rufen mehr Interesse hervor als weniger herausfordernde und erfolgversprechende Tätigkeiten: Im Laufe des Schul- und Berufslebens verlagern sich die wahrgenommenen Interessen in die Richtungen, die optimal herausfordern und erfolgreich bewältigt werden können (Harter 1992, Herber 1998b, Abel \& Tarnai 1998, Lewalter \& Schreyer 2000, Krapp 2002, Lewalter 2002). Umgekehrt fördern Interessen bereichsspezifisch nicht nur Schulleistungen, die Lernmotivation und kognitive Prozesse im Allgemeinen, sondern das Kompetenzund Selbstwirksamkeitsstreben sowie die intrinsische Motivation (Nenniger 1988, Nenniger et al. 1993, Schiefele 1988, 1991, Hidi 1990, Krapp \& Prenzel 1992, Abel \& Tarnai 1998, Herber et al. 2000, Boekaerts \& Boscolo 2002, Ainley et al. 2002, Hidi et al. 2002, Hoffmann 2002, Renninger et al. 2002, Lewalter 2002).

Zusammengefasst: Menschen in allen Entwicklungsstadien ihres Lebens suchen nach Gelegenheiten (herausfordernden Situationen), um ihre Kompetenzen zu entwickeln, auszubauen und aufrecht zu erhalten. Sie entwickeln dabei Gefühle der Selbstwirksamkeit, intrinsische Motivationen und entsprechende Interessen.

\subsubsection{Explorationsstreben}

Berlyne (1960, 1978) zeigte auf, dass durch überraschende, von den eigenen Erwartungen abweichende Ereignisse sowie inkongruente, komplexe Wahrnehmungsobjekte starke Motivationen hervorgerufen werden können, die als (moderate) Herausforderungen an das Kompetenzstreben fungieren. Schon wenige Monate alte Kinder betrachten einen neuen Reiz länger als einen, den sie kurz vorher gesehenen haben (Eliot 2001). Videospiele erzeugen auf diese Weise ein beträchtliches
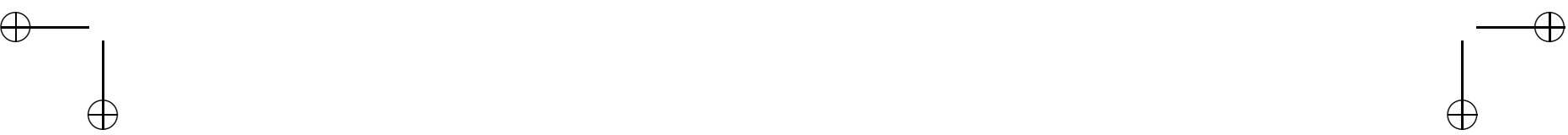


$$
\text { "herber" — 2006/6/26 - 10:27 — page } 6-\# 6
$$

Maß an Abhängigkeit (Herber 2004). Umweltreize, die weder das Neuigkeitsstreben noch mäßige Diskrepanzerlebnisse hervorrufen, werden nicht beachtet. Werden Situationen als zu unbekannt, abweichend vom Vertrauten oder diskrepant zu den eigenen Verarbeitungsmöglichkeiten wahrgenommen, werden sie entweder (abwehrend) ignoriert oder verursachen Angst bis hin zu Panikreaktionen. Die Wechselwirkung von Neugier (Explorationsstreben) und Angst hat auch entscheidenden Einfluss auf die kognitive Bewältungung von Problemlöseaufgaben (z.B. Slusarek 1995).

Die Tendenz, sich besonders mit mittleren Herausforderungslagen auseinander zu setzen, bringt die Forschung des Explorationsstrebens in die Nähe der Kompetenzmotivation: „In the light of White's (1959) provocative work, which sees exploration, curiosity, mastery, play, and competence as expressions of one and the same basic tendency - the urge toward competence or effectance - the significance of such sequence questions is enhanced“ Als da sind: „four components of 'effectance motivation': - 'response variation', 'curiosity for novel stimuli', 'mastery for the sake of competence' and 'preference for challenging tasks'. "Görlitz 1987, 94f.)

Ein neuer Reiz stellt eine Herausforderung dar, fungiert als ungelöstes Problem. Wenn eine Person sich auf ein neues Ereignis oder eine herausfordernde Aufgabe einlässt, versucht sie diese Diskrepanz zwischen neuer und bisheriger Erfahrung, zwischen gegenwärtigem Können und der zu erreichenden Lösungskompetenz zu verringern. Deci (1975) hat dies als ein ständiges Suchen nach passenden Herausforderungen und dementsprechend als permanentes Streben nach Überwindung und Meistern der dabei auftretenden Schwierigkeiten bezeichnet (wenn man nicht in seinen Bemühungen ständig frustriert wurde und daraus gelernte Hilflosigkeit bis depressive Stimmungen erwuchsen, vgl. Seligman 1975/1983, Meyer 2000).

\subsubsection{Internale Attribution}

Gelernte Hilflosigkeit entsteht nicht, wenn eine Person überzeugt ist, dass Lebensereignisse im Allgemeinen von einem selbst verursacht und somit kontrolliert werden können, bei einem (perceived) ,internal locus of control“ die Verstärkung durch die Umwelt von einem selbst machbar erscheint (Rotter 1966, Rotter et al. 1972). Weiner et al. (1971) differenzierten diesen Ansatz im Sinne von Heider (1958/1977): Wenn Personen Erfolg den eigenen Fähigkeiten, Misserfolg aber mangelnder Anstrengung zuschreiben (also jeweils internal attribuieren), wird gelernte Hilflosigkeit eher nicht auftreten. Wenn Misserfolg allerdings - internal - 
auf (stabile) Unfähigkeit, und Erfolg - external - z.B. auf Hilfeleistung durch Fremde (Fremdkontrolle), Zufall, Glück, etc. attribuiert werden, ist mit Misserfolgsfurcht, Resignation, gelernter Hilflosigkeit, „Aus-dem-Felde-Gehen“ (Lewin 1982), etc. zu rechnen (vgl. Weiner 1972, 1996, Herber 1979, 1998a, Herber \& Vásárhelyi 2003, Dresel 2004).

\subsubsection{Selbstbestimmungsmotivation}

Eine wesentliche Wurzel des Kompetenzstrebens stellt das Ringen um Autonomie, Selbstbestimmung, Selbstregulation, etc. dar (DeCharms 1968, 1973, Zimmerman \& Schunk 1989, Maehr \& Pintrich 1991, Deci 1996, Deci \& Ryan 2000, Lewalter \& Schreyer 2000, Kuhl 2001, Krapp 2002, Lewalter 2002, Britt 2003, Gagné 2003, Fröhlich \& Kuhl 2003). Dieses Streben nach Selbstregulation kann ebenso unbewusst wie bewusst verursacht sein und ist auch in seinem Prozessverlauf nicht „, bewusstseinspflichtig“ (z.B. Kuhl 2001, Fröhlich \& Kuhl 2003, Berridge \& Winkielman 2003, Riketta \& Dauenheimer 2003).

Personen suchen normalerweise Aktivitäten auf, die sie nach ihrem eigenen Willen gestalten können (Herber 1998b, Kuhl 2001, Kuhl \& Kazén 2003). Eine wichtige Komponente dieses Willens nach Selbstbestimmung stellt die Fähigkeit zur internalen Ursachenzuschreibung dar (siehe 1.1.5).

Ein- und dieselbe Tätigkeit kann mehr oder weniger motivierend sein, je nachdem ob sie als von einem selbst ausgewählt und passend zu den eigenen Bedürfnissen erlebt wird oder ob sie einem von außen aufgedrängt wird (vgl. Deci et al. 2001, Sheldon 2001, Kasser \& Ryan 2001, Kuhl 2001, Kazén et al. 2003, Herber 2004). Selbst das Lieblingsbuch, die Lieblingsmusik kann einem entfremdet und unangenehm werden, wenn man es - z.B. unter bestimmten didaktischen Gesichtspunkten - analytisch lesen oder hören muss, um damit eine Prüfung zu bestehen, oder wenn man als Versuchsperson - in einem wissenschaftlichen Ansprüchen genügen sollenden „Verhör" - darüber Rechenschaft ablegen muss (vgl. Schleiss 2004).

Besonders wenn man in seinem Handeln einer fremden Kontrolle unterworfen ist (auch wenn dies eine Hilfeleistung impliziert), sinkt die Selbstbestimmungsmotivation und man verliert die Freude an der Sache (Herber 1994).

\subsubsection{Selbstwertstreben und Selbstkonzept}

Menschen streben - neben der Aufrechterhaltung ihres aktuellen Selbstkonzepts - nach Verbesserung ihres Selbstwertgefühls (zur einschlägigen Forschung siehe u.a. Covington 1992, Kuhl, 2001, Crocker \& Park 2003, Herber 2004, Doering 
2004). Dieses impliziert - zumindest in den ersten Entwicklungsstadien - gegenseitig wertschätzende Beziehungen und ist essentiell für die Kompetenzmotivation (diese kann sich ohne positives Selbstwertgefühl nicht entwickeln, vgl. Maslow 1943, 1954/1977, Herber 1972, 1979, Covington 1992, Lewalter \& Schreyer 2000, Schmuck \& Sheldon 2001, Lewalter 2002, Leary \& Tangney 2003, Dippl 2004).

„... humans have a need for competency - the ability to effect outcomes or to master the environment. Mutually caring relationships and the ability to master one's environment both contribute to survival, the overarching and primary goal of humans - what people really need is true relatedness and competence, rather than illusory perceptions of relatedness and competence. Although positive illusions about the self are linked to positive affect, they do not enhance true competency and may incur longterm costs - college students with unrealistic positive views of their academic abilities were initially higher in self-esteem than students who appraised their abilities realistically but that over the course of their college experience they disengaged from academics, and their self-esteem declined. In the long run, peoples's attempts to maintain, enhance, and protect contingent self-esteem may detract from their ability to form and maintain mutually caring relationships and acquire mastery through learning experiences." (Crocker \& Park 2003, 304f.)

Das faustische (erkenntnisgeleitete) Streben nach Selbst- und Welterkenntnis, um ein (umfassend-beglückendes) Selbstwerterleben zu steigern, kann einen hohen Zoll an geistiger und physischer Gesundheit einfordern (vgl. Schneider 1990, Crocker \& Park 2003, Pyszczynski et al. 2003, Herber 2004).

Moderne Selbstkonzeptforschung (z.B. Leary \& Tangney 2003, Schöne et al. 2003, Spinath \& Schöne 2003, Brunstein 2003, Rheinberg 2004) trennt Fähigkeitsselbstkonzepte und Selbstwertkonzepte. Erstere umfassen kognitive Repräsentationen (z.B. über die eigene Intelligenz), letztere die affektiv-bewertenden Prozesse des ganzheitlichen Selbst-Erlebens, das alle partiellen Emotionen und Kognitionen integrierende Selbst-Fühlen (vgl. z.B. Kuhl 2001, Damasio 2003, Herber 2004).

Doch lassen sich Selbstwert- und Selbstkonzeptforschung als emotional-kognitive Interaktionsstruktur konzeptuell verbinden (vgl. Kuhl 2001, Fröhlich \& Kuhl 2003, Aronson et al. 2004, Herber 2004), so dass ein weiter Bereich von selbstbezogenen Phänomenen in integrativer Form abgedeckt werden kann.

Solange sich noch nicht gelernte Hilflosigkeit (Seligman 1975/1983, Meyer 2000), ein negatives Selbstkonzept, Depressivität, etc. verfestigt haben, suchen Menschen einen hohen Grad von Selbstachtung und ein positives Selbstwertgefühl aufrecht zu erhalten. Beides ist bedroht, wenn „kognitive Dissonanz" (Festinger 
1957) auftritt. Diese erzeugt Unbehagen, wenn im Erleben, Denken und Handeln „die Dinge nicht zusammen passen“, wenn z.B. eigene Wünsche, Verhaltensweisen, etc. nicht mit dem Selbstkonzept übereinstimmen. Entweder man ändert sein Verhalten, unterdrückt (peinliche) Wünsche bzw. überformt sie in Richtung des eigenen Selbstkonzepts (Freuds Sublimierung) oder man rechtfertigt im Nachhinein das betreffende Verhalten (Wünschen), indem man sein Selbstkonzept so „verdreht", dass man sich wieder in sich stimmig fühlt, über ein konsistentes Selbstkonzept zu verfügen meint (z.B. durch „Rationalisierung“ im Sinne von Freud 1904-5/1965). So entsteht ein labiles Selbstkonzept und damit eine ständige Bedrohung des Selbstwertgefühls.

Bei Entscheidungen zwischen widersprüchlichen Alternativen überzeugt man im Nachhinein sich selbst von der Richtigkeit der getroffenen Entscheidung, indem man die Vorliebe für die gewählte Alternative „hinaufreguliert“ bzw. für den nicht gewählten Bereich „hinunterreguliert“, besonders wenn man in die gewählte Alternative bereits sehr viel investiert hat. Man kann dann nur schwer zurück, selbst wenn viel Unangenehmes mit dieser Wahl auf einen zukommt und ertragen werden muss (vgl. Brandstätter 1998, Kuhl 2001, Herber \& Vásárhelyi 2002a, Aronson et al. 2004).

Je weniger Rechtfertigung von außen (objektiv-sachlich oder durch soziale Bewertung) für das eigene Wünschen und Handeln zur Verfügung steht, desto mehr muss es „aus sich selbst heraus" aufgewertet werden. So kann aus mangelnder extrinsischer Motivation ein intrinsisches, ,frei“ entschiedenes Bedürfnis entstehen, um die „Summe der Verstärkungen konstant“ zu halten bzw. ein konsistentes Selbstwertgefühl herzustellen (ein Gefühl für ein „faires“ Gleichgewicht, einen fairen Ausgleich). Dazu gehört im umgekehrten Sinne auch, dass man zusätzliche sachliche und soziale Anerkennung für einen von Versagensangst, sozialer Zurückweisung, etc. bedrohten Anteil des eigenen Selbst sucht. Dies wird zum Problem, wenn man an diesem Überkompensationsmechanismus (sensu Adler 1966) fixiert bleibt und damit zu viel Energie bindet, die man besser für die Entfaltung der eigenen Persönlichkeit nützen sollte (für Selbstermöglichung und -verwirklichung im Sinne von Herber 2004).

\subsubsection{Erfolgsmotivation (und Misserfolgsmotivation)}

Den theoretisch und methodologisch fundiertesten Beitrag zur Aufhellung der mit dem Terminus „Kompetenzstreben“ zusammengefassten Motivationskonfiguration liefert die über fünfzigjährige (paradigmenverschmelzende) Forschung auf dem Gebiete der Leistungsmotivation (vgl. McClelland et al. 1953, Atkinson \& 
"herber" — 2006/6/26 - 10:27 — page $10-\# 10$

Feather 1966, Herber 1972, 1976, 1979, Moulton 1974, McClelland 1984, 1987, Weiner 1996, Herber et al. 1999, 2001, Kuhl 2001, Rheinberg 2002, 2004, Herber \& Vásárhelyi 2002b, 2003, Schmalt 2003).

Mit Atkinson (1957) beginnt die theoretische (mittels mathematischer Modellbildung) und empirische Erforschung von Erfolgs- und Misserfolgsmotivation, die in der Dynamischen Handlungstheorie sowohl Prinzipien einer allgemeinen Motivationstheorie (Atkinson \& Birch 1970, Atkinson 1992) als auch spezifizierte Annahmen für die Leistungsmotivation (LM) generiert (Atkinson \& Birch 1974). Das Grundproblem dieser mathematischen Formulierungen besteht in der mangelnden Festlegung des zulässigen Wertebereichs der Motivausprägungen, denn erst durch solche Normierungen werden empirische Ergebnisse untereinander vergleichbar (siehe Herber \& Vásárhelyi 2003). Ähnliche Probleme ergeben sich bei der mathematischen Modellierung der die LM-Forschung fundierenden Anspruchsniveauforschung von Lewin et al. (1944) durch Kuhl $(1986,2001)$ und (,natürlich“) bei der „Messung“ des LM mittels TAT (z.B. Langens \& Schüler 2003, Rheinberg 2004).

Atkinson konzipierte das Leistungsverhalten als Resultante eines Konflikts zwischen einer erfolgsmotivierten Handlungstendenz und einer misserfolgsängstlichen Vermeidungstendenz. Leistungssituationen aufzusuchen setzt Hoffnung auf Erfolg voraus, ein (bereichsspezifisch) relativ stabiles Motiv, das „nicht bewusstseinspflichtig" ist (Kuhl 2001). Dieses Motiv wird mittels Fantasiegeschichten gemessen, die bei den Versuchspersonen mit mehrdeutigen Bildern provoziert werden und mehr oder weniger bewusste motivrelevante Inhalte zum Ausdruck bringen. Die testtheoretischen Eigenschaften hinsichtlich Validität und Reliabilität wurden von den ersten Durchführungs- und Auswertungsrichtlinien bei McClelland et al. (1953) über die grundlegenden Weiterentwicklungen bei Heckhausen (1963), Atkinson (1992), McClelland \& Koestner (1992) und vor allem Kuhl et al. (2003) sowie Scheffer et al. (2003) so verbessert, dass heute von einer vergleichbaren Reliabilität wie bei Fragebogenverfahren und einer diesen überlegenen Konstruktvalidität ausgegangen werden kann (zu grundlegenden Fragen der Motivationsdiagnostik siehe auch Heckhausen 1989, McClelland et al. 1992, Kuhl 2001, Stiensmeier-Pelster \& Rheinberg 2003, Scheffer 2003, Herber \& Vásárhelyi 2003, Rheinberg 2002, 2004).

Das Misserfolgsmeidungsmotiv ist darauf gerichtet, die mit Misserfolg einhergehende Beschämung und Beeinträchtigung des Selbstwertgefühls zu vermeiden, indem mehr oder weniger bewusst Leistungssituationen (Herausforderungen an 
die eigene Tüchtigkeit) gemieden oder - z.B. durch ein extrem hohes Anspruchsniveau - im Sinne von Adler (1966) überkompensiert werden (vgl. Herber 1972, McClelland \& Watson 1973, McClelland \& Teague 1975).

Leistungsbezogene Anreize bzw. Aktivitäten können als Herausforderungen an das eigene Kompetenzstreben interpretiert werden: Immer werden durch Probleme/Aufgaben sowohl positive als auch negative Emotionen als mögliche Handlungsausgänge antizipiert und damit kompetenzstärkende bzw. -mindernde Konsequenzen mit einer entsprechenden Hebung oder Senkung des Selbstwertgefühls verbunden (vgl. McClelland et al. 1953, Herber 1972, Heckhausen 1977, McClelland 1987, Covington 1992, Dippl 2004).

Wenn Erzieher Kinder und Jugendliche ermutigen, leistungsbezogene Aufgaben in selbständiger Weise zu lösen, ihre Kompetenzen auszuprobieren und (allmählich) zu steigern, steigt die Wahrscheinlichkeit (bereichsspezifisch) erfolgsmotiviert zu werden. Wenn Erzieher aber das Kompetenzstreben ihrer Zöglinge behindern oder unterdrücken, indem selbstgesteuertes Lösen von Aufgaben/Problemen nicht zugelassen wird (sondern nur das Befolgen und Kopieren der Vorgaben durch die Erzieher), entsteht leistungsvermeidende Misserfolgsfurcht, um das eigene Kompetenz- und Selbstwertgefühl nicht zu gefährden (Leistungsvermeidung dient der Abwehr von selbstwertschädlichen Beeinträchtigungen des Kompetenzstrebens, das so wenigsten illusionär aufrechterhalten werden kann: Was man gar nicht versucht, kann auch nicht missglücken. Eine überkompensierende Coping-Strategie von Leistungsangst zeigt sich in übersteigerten Zielsetzungsfantasien bei einem Teil der Misserfolgsmotivierten). Starker Leistungsdruck spricht hochleistungs- und erfolgsmotivierte Menschen nicht an (besonders wenn Leistung von außen normativ eingefordert wird). Personen mit einem starken, erfolgsorientierten TAT-Leistungsmotiv (gemessen mittels Fantasiegeschichten zu mehrdeutigen Bildvorlagen) versuchen mehr als Personen mit einem niedrigen und/oder misserfolgsorientierten LM eigene Fähigkeiten auf selbstbestimmte Weise zu entdecken, zu entwickeln und in entsprechende Handlungskompetenzen (Leistungen) zu transformieren. Dabei versuchen sie ständig, ihr eigenes Leistungsniveau zu steigern oder wenigstens nicht absinken zu lassen (vgl. z.B. McClelland 1953, Herber 1979, Herber \& Vásárhelyi 2003, Langens \& Schüler 2003).

Erfolgshoffnung und Misserfolgsfurcht sind im Sinne von Kuhl (2001) nicht bewusstseinspflichtig. McClelland et al. (1992) kennzeichnen sie als „implizite“ Motive (im Unterschied zu expliziten, bewussten Werthaltungen). Auch bei Atkinson $(1957,1992)$ geschieht das Zu- und Abnehmen von Motivstärken (bzw. der 
"herber" — 2006/6/26 — 10:27 — page 12 — \#12

Wechsel von Motiven, z.B. zwischen Leistungs- und Sozialmotivation) eher unbewusst (im Unterschied zu kognitiv kalibrierten Einschätzungen der Schwierigkeit und damit der (Miss-)Erfolgswahrscheinlichkeit spezifischer Aufgaben). Der emotionale Anreiz einer Aufgabe hängt bei Atkinson von ihrer eingeschätzten Erfolgswahrscheinlichkeit ab: Je leichter eine Aufgabe eingeschätzt wird, desto weniger Anreiz für das eigene Kompetenzstreben stellt sie dar, umso bedrohlicher aber erscheint ein eventueller Misserfolg. Je schwieriger eine Leistungsanforderung erscheint, desto höher ist ihr Anreiz in Bezug auf das Kompetenzstreben und umso geringer ist die Beschämung (Beeinträchtigung) bei einem erwartbaren Misserfolg. Eine beliebte Abwehrstrategie, um das eigene Kompetenz- und Selbstwertgefühl - zumindest situativ - nicht zu schädigen, ist daher die (überkompensatorische) Wahl eines extrem hohen Schwierigkeitsgrades (den sich sonst niemand zutraut) bei gleichzeitiger Minimierung der eigenen Anstrengung (vgl. Adler 1966, Covington 1992, Dresel 2004). Objektiv bedeutet dies allerdings langfristig eine Schädigung der realen Kompetenzen und die Verstärkung irrealer Tendenzen, sein Kompetenz- und Selbstwertgefühl hochzuhalten (indem dieses durch die Vermeidung von Leistungsanforderungen nicht in Frage gestellt wird, was der theoretisch und empirisch belegten Charakteristik von Misserfolgsmotivieren entspricht, die entweder Leistungssituationen fliehen oder durch Überkompensation ihrer Misserfolgsangst realitätsfremden Wunschzielen nachträumen).

\subsection{Kompetenzerwerb im kognitiven Bereich}

Durch einen unabsehbaren Korpus an Literatur kann der Einfluss von kognitiven Strategien auf den Kompetenzerwerb in schulisch-akademischen Bereichen aufgezeigt werden (z.B. Neber 1981, 1987, Schleider 1993, van Oers 1996, Gustafsson \& Undheim 1996, Lukesch 1998, van der Meer 1998, Astleitner 1998, Schaeken et al. 2000, Seel 2000, Torff \& Sternberg 2001, Lukesch 2001, Sternberg \& Zhang 2001, Astleitner et al. 2002), unter immer stärkerer Einbeziehung der neuen Informationstechnologien (z.B. Klahr et al. 1987, Lehrer 1989, Greif 1996, Seidler 1997, Astleitner 1997, 2003a,b, Vásárhelyi 1999, Dörner 1999, Wolfram 2002, Farahat \& Astleitner 2004, Priemer \& Schön 2004).

Im Folgenden fassen wir wichtige Ergebnisse der kognitionspsychologischen Forschung samt didaktischen Anwendungsstrategien zusammen (Herber 1983, 1984, 1998c, Vásárhelyi 1996, 2004a,b).

Kompetentes Denken im akademischen Zusammenhang erfolgt zielgerichtetbewusst in mehreren Phasen:
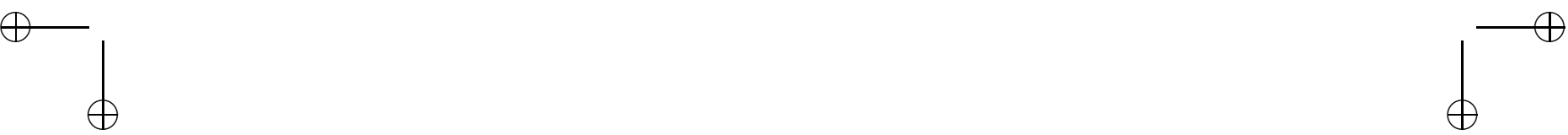
"herber" — 2006/6/26 — 10:27 — page 13 — \#13

$\underline{\text { Kompetenzstreben und Kompetenzerwerb: Funktionale didaktische Fördermöglichkeiten... } 13}$

(1) Am Beginn stehen Suchprozesse, in denen Ziele und entsprechende Kriterien elaboriert werden, welche die Lösung eines Problems anzeigen.

(2) Ist die Zieldomäne formuliert, geht es darum, verschiedene (alternative) Möglichkeiten der Zielerreichung zu konzipieren und auf Basis des vorhandenen und noch zu erwerbenden Wissens/Könnens gegeneinander abzuwägen.

Die Suchprozesse werden oft im vorhandenen Quellbereich der Person durchgeführt (im Langzeitgedächtnis mit den gespeicherten kognitiven Strukturen, Fakten, Problemlösestrategien, etc.). Darüber hinaus können externe Quellen angezapft werden (das Wissen/Können anderer Personen, traditionelle oder elektronische Lexika, etc.). Andere reale oder virtuelle Personen können als Modelle für die (geeignete) Anwendung von Problemlösestrategien dienen.

Kompetentes Denken versucht auch eine vernünftige Relation zwischen Kostenaufwand (Zeit, Energie, etc.) und der angestrebten Lösungsgüte herzustellen. Im idealen Fall hingegen werden alle Lösungsmöglichkeiten in Betracht gezogen und rational (ohne emotionale positive oder negative „Fixierungen“) miteinander verglichen und dann erst (evtl. kreativ kombiniert) zur Anwendung gebracht.

Kompetentes Denken stellt - in Einschränkung idealisierter Gütestandards eine individuelle optimale Strategie dar. Diese basiert auf einem idiosynkratischen System von Wissen (Fakten, Begriffen, Strukturen, Strategien) sowie auf einer personspezifischen kognitiven wie emotional-motivationalen Problemlösekapazität (z.B. Intelligenz, Kreativität, Leistungsmotivation, positive oder negative emotionale [Grund-]Befindlichkeit, z.B. Stabilität [bis Rigidität] oder Dynamik [bis Labilität] im positiven bzw. negativen Erleben und Bewerten von gewissen Leistungssituationen).

Entsprechend der - individuell verankerten - kognitiven, emotionalen und motivationalen Unterschiede werden Kosten und Nutzen der in Betracht gezogenen Problemlöseprozesse eingeschätzt. Eine individuell optimal erscheinende Lösung muss daher nicht „objektiv“ (logisch-rational) optimal sein (vgl. die vielfältigen Forschungen zu individuellen „,belief-systems“, z.B. Hogarth \& Einhorn 1992, Elio \& Pelletier 1997, Politzer \& Carles 2001, Dieussaert et al. 2000, 2002). Solche Abweichungen der individuellen von objektiv optimalen Lösungsstrategien kennzeichnen unterschiedliche kognitive Stile (z.B. Riding 2001, Entwistle et al. 2001). So kann ein impulsiver Reaktionsstil übermäßig viel Energie in nicht geeigneten Lösungsbemühungen „verschleudern“ und zu wenig abwägende Reflexivität von angestrebten Zielen und Lösungswegen wie auch ein zu geringes Ausmaß an Selbstkritik bei der Fehlersuche mit sich bringen.
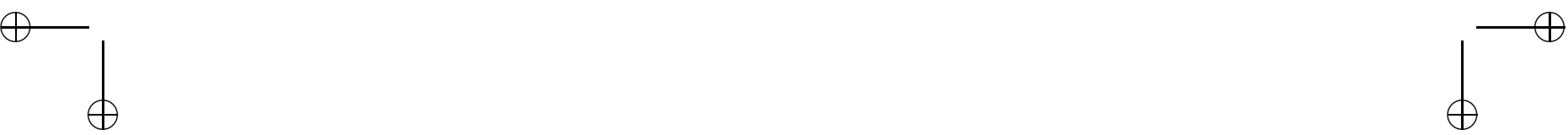
"herber" — 2006/6/26 — 10:27 — page 14 — \#14

Ein systematisch angelegtes didaktisches Vorgehen wird sowohl Anknüpfungsmöglichkeiten an unterschiedliche kognitive Stile des Kompetenzerwerbs als auch an individuelle Motivationskonfigurationen des Kompetenzstrebens in Form weitgehender selbstdiagnostischer Erprobungen der individuellen Optimierungsstrategien ermöglichen sowie behutsam lenkend eine günstige Weiterentwicklung des individuellen Interaktionssystems in kognitiver, emotionaler und motivationaler Hinsicht anstreben. Maßnahmen der Inneren Differenzierung und Individualisierung scheinen uns dafür besonders geeignet zu sein.

1.3. Wechselwirkungen von kognitiven und emotional-motivationalen Prozessen

Die empirische Forschung über den Zusammenhang von Kompetenzstreben und kognitivem Kompetenzerwerb gibt einerseits Anlass zur Skepsis, ob Verbesserungen der Motivationslage auch tatsächlich entsprechende Verbesserungen der kognitiven Fähigkeiten mit sich bringen (z.B. Herber 1987, Herber et al. 2000, Fuchs et al. 2001, Herber \& Vásárhelyi 2003), andererseits sind emotions- und motivationsbasierte Effekte auf kognitive Lernleistungen (vice versa) in differenzierter, vielfältiger Weise nachgewiesen worden (z.B. Yerkes \& Dodson 1908, McClelland et al. 1953, Moulton 1974, Karabenik \& Youssef 1974, Horner 1974a,b, Raynor 1974, Raynor \& Rubin 1974, Atkinson 1974a,b, Brown 1974, Weiner 1974a,b, Cooper et al. 1978, Herber et al. 1978, Atkinson \& Lens 1980, Eysenck 1982, Schiefele 1988, 1991, McLeod, \& Adams 1989, Schneider \& Weinert 1990, Christianson 1992, Jonassen \& Grabowski 1993, Nenniger et al. 1993, Shaw \& Runco 1994, Schutz 1994, Spies 1995, Süß 1996, Astleitner 1997, Herber et al. 1998e, 2000, Wegge 1998, Spies \& Lüer 1998, Satow \& Schwarzer 2000, Kuhl 2001, Leutner et al. 2001, Lewalter 1997, 2002, Stiensmeier-Pelster \& Rheinberg 2003, Damasio 2003, Drese 2004, Rheinberg 2002, 2004). Die wesentliche Aussage dieser Untersuchungen besteht darin, dass eine mittlere Aktivierung durch hohe Erfolgsmotivation und wenig Furcht vor Misserfolg (bezogen auf kürzere oder längere Planungshorizonte), hohe intrinsische Motivation und sachbezogene Interessen (wenig gestört durch extrinsische Motivationen), hohes Kompetenzstreben u.a. leistungsbezogene Motivationen (mittlere Risikobereitschaft, Toleranz für Abweichungen vom Erwarteten, Aushalten von Unsicherheit und Mehrdeutigkeit, etc.) insgesamt zu besseren kognitiven Leistungen führt als zu wenig oder zu hohe Erregung und Aktivierung, hervorgerufen durch geringen oder übermäßigen Anreiz bzw. durch zu hohe, langanhaltende Ängste im Falle von (verkrampfter, regressiv-rigider) 
Übermotivierung oder durch selbstentfremdende Aktivierung „, von außen“. „Herabregulierung" negativen Affekts, verbunden mit „Hinaufregulierung“ positiven Affekts auf ein moderates, selbstgesteuertes, ganzheitlich-integrierendes, positiv getöntes, doch analytisch kontrolliertes emotional-kognitives Informationsverarbeitungsniveau sensu Kuhl (2001) könnte als Ansatz einer systemischen (auch neurophysiologisch fundierten) funktionalen Erklärung für die zahlreichen, einander stützenden Befunde heterogener Provenienz herangezogen werden.

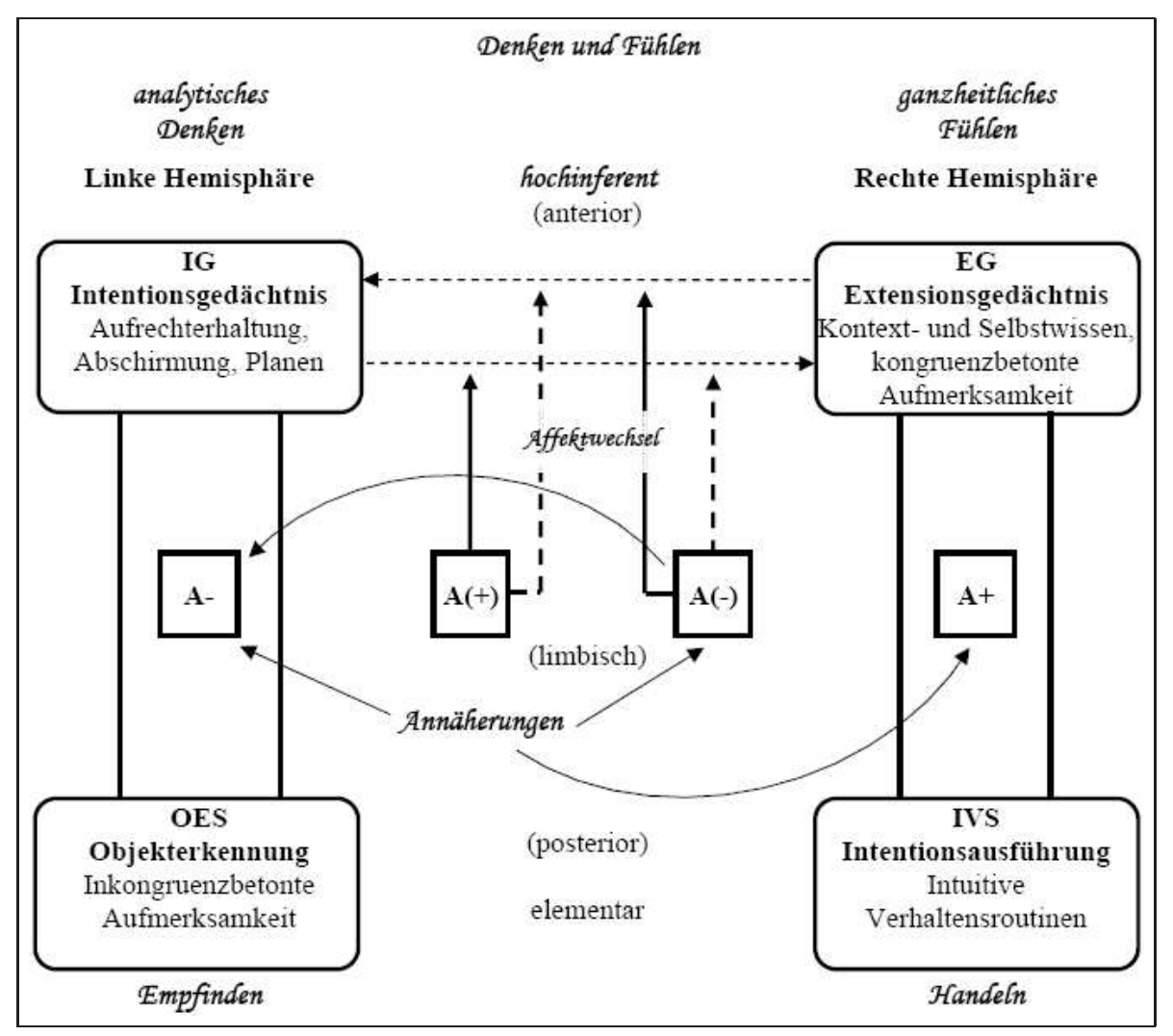

Abbildung 1. System-Interaktionen als Austausch zwischen linker und rechter Hemisphäre (vertikale Doppellinien symbolisieren den affektunabhängigen ipsilateralen Austausch; gestrichelte Pfeile Hemmungsrelationen; durchgezogene Pfeile $=$ Bahnungsrelationen; $A(+)=$ Herabregulierung von positivem Affekt; $A(-)=$ Herabregulierung von negativem Affekt): Nur der Affektwechsel schafft kontralaterale Systemverbindungen (nach Kuhl 2001, 685). 
"herber" — 2006/6/26 — 10:27 — page 16 — \#16

Dazu folgendes Schema nach Kuhl (2001, 685, mit interpretativen Ergänzungen, siehe Abbildung 1). Die wesentliche Aussage ist folgende: Wenn zwischen subjektiver Erwartung (auf Grund bisheriger Erfahrungen) und aktueller Wahrnehmung deutliche Inkongruenzen empfunden werden, entsteht negativer Affekt (Verunsicherung, Angst) und analytisches Denken setzt ein, verbunden mit der Suche nach entsprechenden Zielvorstellungen (Intentionsgedächtnis), um das entstandene Problem zu lösen. Gelingen (wenigstens) Teilerfolge in diesem analytisch-sequentiellen Modus, kann der negative Affekt so weit herabreguliert werden, dass bei zunehmender Problemlösung die kognitive Schemaoptimierung (Akkomodation nach Piaget 1980) in das weitgespannte Netzwerk der selbstrelevanten Wissens integriert werden kann (eine erfolgreiche Problemlösung wird vom Selbst angenommen, bereichert Selbstkonzept und Selbstwertgefühl).

Kommt man mit dem analytisch-sequentiellen Modus nicht voran, ist es notwendig, sich von diesem Modus zu lösen (das Problem kurzfristig zu „vergessen“) und sich seiner selbst fühlend zu vergewissern (Kongruenz mit sich selbst herzustellen - etwa durch Anknüpfung an das biografische Extensionsgedächtnis, an alle positiv durchlebten und „gemeisterten" Situationen).

Dies bewirkt ein „Umschalten“ auf positiv besetztes „Selbstwissen“, was umso leichter gelingt, je mehr Teilerfolge im analytischen Modus erreicht werden konnten (weil dann die linkshemisphärische negative Affektlage schon so weit herabreguliert wurde, dass bei mittlerer Affektlage - über den „Balken“ - die rechte Hemisphäre zugeschaltet werden kann). Damit tut sich mit dem Extensionsgedächtnis ein weiter (emotional positiv getönter) Suchraum auf, der kreatives Umstrukturieren und damit entsprechende Lösungsalternativen ermöglicht (man ist wieder - flexibel - handlungsfähig geworden). Erst wenn erfolgreiche Problemlösestrategien „Selbstkonzepte“ im Sinne von Kohut (1977/1979) geworden sind, also in das selbstrelevante Extensionsgedächtnis integriert werden können, werden intuitive Verhaltensroutinen ausgebildet, die das erfolgreiche Handeln „selbst"-verständlich steuern.

2. Förderung von Kompetenzstreben und Kompetenzerwerb durch Maßnahmen der Inneren Differenzierung (I. D.) und Individualisierung im Unterricht

Als didaktische und erzieherische Maxime des von uns entwickelten Modells der I. D. kann gelten, dass der Lernende dort abgeholt wird, wo er hinsichtlich 
seiner kognitiven und motivationalen Kompetenzen „steht“ und dort hingeführt werden soll, wo er - seinen Möglichkeiten nach - sein könnte.

Das im Praxiszusammenhang des schulischen Klassenunterrichts entwickelte Grundmodell (Herber 1983) samt empirischen Überprüfungen (Herber 1988d) versucht einen Unterrichtsprozess zu strukturieren, der einerseits einen „sicheren Pfad" zur Zielerreichung anbietet, andererseits ein flexibles Abweichen und Zurückkehren zu diesem Pfad ermöglicht - mit weitgehend selbst gewählten Schwierigkeitsgraden und inhaltlichen Anknüpfungsmöglichkeiten aus einem vorgegebenen Aufgabenpool, wobei Schüler selbst im Sinne des Lösungs-Rationale für sich und andere Schüler Aufgaben auch selbst konstruieren (erfinden) dürfen. Die Sozialform ist ebenfalls weitgehend frei wählbar, die Hilfestellung durch Lehrer und Mitschüler wird vom einzelnen Schüler aus initiiert (und nicht „von außen“ aufgedrängt).

Weiters kann - in Weiterentwicklung des Grundmodells - zwischen traditioneller und elektronischer Hilfe selbstgesteuert gewählt werden (vgl. Fuchs et al. 2001, Herber \& Vásárhelyi 2003, Vásárhelyi 2004a), wobei auch außerschulische Lernumgebungen im Sinne der I. D. strukturiert werden können (Vásárhelyi 2004c).

Im Folgenden werden Auswirkungen der bisher dargestellten theoretischen Positionen auf Maßnahmen der I. D. diskutiert. In diesem Zusammenhang ist uns folgender Aspekt besonders wichtig: Das Lösen derselben Aufgabe kann während des (nach Aufbau, Einbettung, Hilfestellung, Werkzeugangebot, Ausmaß des Lernschrittes, etc.) differenzierten/individualisierten Lehr-, Lernprozesses den Kompetenzerwerb auf unterschiedlichen Bereichen und Stufen fördern (vgl. Ambrus 2003). Dieses zu zeigen, wird Aufgabe des 3. Kapitels dieses Artikels sein. Derselbe Inhalt kann durch analog aufgebaute Strukturen angeboten, entsprechende Lehr/Lernziele können auf unterschiedlichen Wegen erreicht werden. Was für einen bestimmten Lerner eine befriedigende Lösung darstellt (etwa als Fundamentumsziel), ist für einen anderen eine Zwischenstufe zu einem vertieften oder erweiterten Verständnis (etwa im Sinne eines Additumsziels). Dabei können - grundsätzlich - elektronische wahlweise mit traditionellen Arbeitsblättern kombiniert werden.

\section{Ad 1.1.1 Selbstwirksamkeit}

Diese wird gefördert, wenn jedem Lernenden ermöglicht wird, seine individuelle Selbstwirksamkeit zu erproben, abzusichern und auszubauen. Das bedeutet einerseits maximale Freiheit bei der Aufgabenwahl (bezogen auf gleiche Lernziele) und bei dem Lösungsweg, andererseits individualisierte Hilfenahme (Stufen 


$$
\text { "herber" — 2006/6/26 — 10:27 — page } 18 \text { — \#18 }
$$

der Hilfe, wie Kontrollstellen, anregende Fragen, Starthilfen, Erinnerungshilfen, Lösungsskizzen, detaillierte Lösungen und dosierte didaktische Interventionen, vgl. unten Kap. 3).

Was die (übliche) Beurteilungsform in Schulklassen nach der sozialer Bezugsnorm (Heckhausen 1974) betrifft: Das Selbstwirksamkeitsstreben wird zumindest bei jenen Schülern permanent frustriert und in „gelernte Hilflosigkeit“ (Seligman 1975, Meyer 2000) umgewandelt, die - unter dem Klassendurchschnitt liegend trotz intensiver Bemühungen kaum einmal eine (über-)durchschnittliche Leistung erbringen können.

\section{Ad 1.1.2 Optimale Herausforderung}

Was eine optimale („mittlere“) Herausforderung darstellt, ist nur personspezifisch entscheidbar. Im Modell der I. D. nach Herber \& Vásárhelyi (2003) wird dabei ein Pool von Aufgaben angeboten, die alle als äquivalente (analoge) Modelle einer spezifischen Problemlösestrategie konzipiert sind, sich aber hinsichtlich der "Verarbeitungstiefe“, des Schwierigkeitsgrades, der thematischen Einbettung, etc. unterscheiden (siehe wieder unten Kap. 3).

Grundsätzlich sollten Fundamentumsaufgaben (die von allen Schülern gelöst werden müssen) prototypische Exemplare darstellen, deren Schwierigkeitsgrad so gering wie möglich gehalten werden (vgl. Vásárhelyi 2004a,b). Sie stellen zentrale "Ankerbeispiele" dar, die möglichst viele Merkmale, Relationen, Strukturen, etc. mit allen anderen (potentiellen) Aufgabenstellungen des jeweiligen Lösungsrationales teilen. Additumsaufgaben können sowohl zusätzliche (wissenschaftsbezogene) Vertiefungs- und Erweiterungsaspekte als auch erhöhte Schwierigkeits- und Komplexitätsgrade enthalten. Stellen Fundamentumsaufgaben optimale Herausforderungslagen für eine prototypische Allgemeinbildung dar, führen Additumsaufgaben gezielt zur wissenschaftsbezogenen Forschung hin (mit einem entsprechenden Anspruchsniveau im Sinne von Lewin et al. 1944, vgl. dazu Herber \& Vásárhelyi 2002a).

\section{Ad 1.1.3 Intrinsische Motivation}

Um die intrinsische Motivation zu stärken, sollten möglichst wenige extrinsische (sachfremde) Bewertungen vorgenommen werden (soziales Lob, personbezogener Tadel, Zensuren, Vergleiche mit den Leistungen anderer, etc.) Sachbezogene Rückmeldungen hingegen können die intrinsische Motivation verstärken, z.B. „Dieses ist gelungen, da sind Sie auf dem richtigen Weg, hier aber steckt 


$$
\text { "herber" — 2006/6/26 — 10:27 — page } 19 \text { — \#19 }
$$

Kompetenzstreben und Kompetenzerwerb: Funktionale didaktische Fördermöglichkeiten. . 19

noch ein ungelöstes Problem, vielleicht sollten Sie dieses oder jenes noch mehr beachten ..."

Intrinsisch motivierte Tätigkeiten stärken die Selbstkompetenz und Selbststeuerungsmotivation. Aufgaben sollten selbst aus dem angebotenen Aufgabenpool ausgewählt (bei Beachtung sachlogisch notwendiger Sequenzen) oder auch selbst erfunden/konstruiert werden, um anderen Schülern angeboten werden zu können (diese Form von extrinsischer Motivation kann die intrinsische Motivation durchaus stützen). So entstehen langanhaltende Interessen, die kumulativ zu ständiger Leistungsverbesserung führen können (z.B. Atkinson 1974b, Atkinson \& Lens 1980, Lewalter 2002).

Rückmeldungen im Lernprozess sollten sparsame sachbezogene Verstärkungen und allenfalls minimale Hilfestellungen (didaktische Lernhilfen) enthalten. Zu wissen, auf „dem richtigen Weg“ zu sein bzw. rechtzeitig über eine fehlerhafte Strategie Aufklärung zu erhalten, stärkt durchaus die intrinsische Motivation (vgl. 1.1.7).

\section{Ad 1.1.4 Explorationsstreben}

Neugier und Explorationsstreben werden frustriert und reduziert, wenn Lehrer neuen Stoff ausschließlich vortragen - als gegebenes Wissen, das Schüler sich einfach anzueignen haben. Elaborierte Wissenstrukturen sollten als (erprobte) Problemlösestrategien verstanden werden. Dazu ist es hilfreich, in didaktisch geeigneter Transformation die ursprünglich gegebene Problemlage zu rekonstruieren (Copei 1960), quasi sich in die Lage der Forscher zu versetzen, als sie darangingen, ein wahrgenommenes Problem zu lösen. Minimale Lernhilfen (individualisiert abgestimmte Hinweisreize) sollten weitgehend ausreichen, um das Explorationsstreben in Gang zu halten. Didaktische „Black boxes“ (eventuell elektronisch aufbereitet) könnten sachlogisch geordnet helfen: Man begegnet an der Oberfläche einem Problem. Kann man es lösen, bedarf es weiter keiner didaktischen Hilfe. Wenn man es nicht lösen kann, wird die Oberflächenschale (z.B. bei physikalischen Modellen) entfernt und man bekommt erste Hinweisreize (eventuell rückgekoppelt an bisherige Lösungsversuche, vgl. Vásárhelyi 2004c). Reichen diese Hinweise noch immer nicht aus, werden Schritt für Schritt didaktische Schalen (Problemrepräsentationen) entfernt, bis die gesamte Problemlösestrategie offen gelegt wird. So wird die Neugier auf die nächste Information angestachelt und das Explorationsstreben bleibt - anspruchsvoll - erhalten. 


$$
\text { "herber" — 2006/6/26 — 10:27 — page } 20 \text { — \#20 }
$$

\section{Ad 1.1.5 Internale Attribution}

Die günstigste Strategie scheint im Allgemeinen zu sein, Erfolg den eigenen Fähigkeiten (Begabungen) verbunden mit ausreichender Anstrengung, Misserfolg aber auf mangelnde Anstrengung zurückzuführen. Liegt maximale Anstrengung vor, ist ein Ausweichen auf externale Ursachen günstig (Zufall, Pech, besondere Strenge der Beurteilung bei gewissen Lehrern, Beurteiler mögen einen persönlich nicht, schlechte soziale Beziehungen in einem bestimmten Lehr-/Lernsetting, etc.).

\section{Ad 1.1.6 Selbstbestimmungsmotivation}

Unser Modell der I. D. (Herber \& Vásárhelyi 2002b, Vásárhelyi 2004a,c) bietet zahlreiche Möglichkeiten zur selbstgesteuerten Vorgangsweise an: Schüler können - innerhalb eines sachlogisch sinnvolle Aufbaus - ihr „Aufgabenmenü“ selbst zusammenstellen, (z.B. aufbauend auf ein notwendiges Ausmaß prototypischer Fundamentumsaufgaben in freier Weise die vom Lehrer vorgeschlagene Sequenz von Aktivitäten ändern, Fundamentums- und Additumsaufgaben aus einem vorgegebenen Aufgabenpool mit unterschiedlichen Schwierigkeitsgraden wählen, thematische Querverbindungen aufgreifen, etc., vgl. unten Kap. 3).

Die Individualisierung des Kompetenzerwerbes kann bei komplexeren Sachverhalten dadurch verstärkt werden, dass die Lernenden die persönlich aktuelle Tiefe der Verarbeitung selber bestimmen dürfen. Die Arbeit kann in freier Wahl der Aufgaben und der Reihenfolge der Erarbeitungsmodi mit Hilfe von traditionellen Veranschauungsmitteln (um konkrete Fälle selber untersuchen, Erfahrungen haptisch sammeln, das Allgemeine an konkreten Fällen erproben bzw. erleben zu können) und/oder mit elektronischen Medien, Computeranimationen (um Gesetzmäßigkeiten entdecken, das Situationsspezifische vom Allgemeinen trennen zu können) erfolgen, wobei die Tätigkeit der Schüler durch „paper \& pencil“Arbeitsblätter moderiert bzw. protokolliert wird.

Als erste Erprobung ihrer auf diese Weise erworbenen Kompetenz dürfen die Lernenden selbst Aufgaben konstruieren (diese selbst konstruierten Aufgaben sollen nicht nur für Mitschüler, sondern auch für den Lehrer zur Verfügung gestellt werden und dienen somit als Indikatoren der individuellen Verarbeitungstiefe). 


\section{Ad 1.1.7 Selbstwertstreben und Selbstkonzept}

Menschen lassen sich normaler Weise nur auf sach- oder sozialbezogene Tätigkeiten ein, wenn sie damit ein positives Selbstwertgefühl bzw. Selbstkonzept steigern oder aufrechterhalten können. Werden sie - wie dies im schulischen Unterricht nicht selten der Fall ist - permanent zu Tätigkeiten angehalten, die ihr positives Selbstwertgefühl in Frage stellen oder bedrohen, dann treten Abwehrmechanismen auf den Plan, um Bedrohungen von Selbstwertgefühl und Selbstkonzept hintan zu halten. Beispiel: Ein Schüler bemüht sich redlich, möglichst gute Schulleistungen zu erbringen. Im Vergleich zu seinen früheren Leistungen (individueller Gütemaßstab sensu Heckhausen 1974¹) kommt er tatsächlich voran, seine Leistungen werden objektiv besser. Doch im Vergleichen zu seinen Mitschülern (sozialer Gütemaßstab) kann er sich nicht oder kaum verbessern (sein Rangplatz in der Klasse ist weiterhin - sagen wir - im unteren Drittel und er kommt darüber nicht hinaus). Damit setzt ein verheerender „circulus vitiosus“ ein (vgl. Covington 1992, Dresel 2004): Um sein Selbstwertgefühl zu verteidigen, strengt sich der Schüler nicht mehr an, damit er vor sich selbst und den anderen die Illusion aufrecht erhalten kann, dass er bessere Leistungen erbringen könnte, wenn er sich nur „richtig“ anstrengen würde. Die Sache aber sei es nicht wert, interessiere ihn zu wenig, es stehe nicht dafür, sich dafür anzustrengen (vgl. die Äsop-Fabel: „Die Trauben sind mir zu sauer!“, denkt der Fuchs, nachdem er vergeblich versucht hatte sie zu erreichen, weil sie für ihn einfach zu hoch hingen).

Das Tückische an dieser Abwehrstrategie besteht darin, dass bei fehlender Anstrengung die Leistungen objektiv schlechter werden und dass der Schüler vom Lehrer immer negativer sanktioniert wird - einerseits der schlechter werdenden Leistungen wegen, andererseits aus „moralischen“ Gründen wegen der fehlenden Leistungsbereitschaft („dumm und faul“). Langfristig sinkt damit sein Ansehen auch bei den anderen Schüler (da „Genieblitze" bei fortgesetzter niedriger Anstrengung immer unwahrscheinlicher werden). So verliert der Schüler die soziale Stützung seines Selbstkonzepts und Selbstwertgefühls, wenn er - von Fall zu Fall - Misserfolg mit mangelnder Anstrengung „wegerklären“ will.

Schülern ihren Lernfortschritt zu dokumentieren, indem man sie mit ihren eigenen (früheren) Leistungen vergleicht, kann hier abhelfen (vgl. Rheinberg 1980). Dazu ist es aber notwendig, auf einen sozialen Leistungsvergleich mittels Notenskala zu verzichten (weil dadurch unser Schüler immer in der „schlechteren“ Hälfte

${ }^{1}$ Bezüglich neuerer Forschung zu Bezugsnormen der Leistungsevaluierung siehe Dickhäuser \& Rheinberg 2003. 
"herber" — 2006/6/26 — 10:27 — page 22 — \#22

unter dem Durchschnitt platziert wird, egal welche individuellen Fortschritte er realisiert). Sachbezogene (lernziel- und aufgabenbezogene) Rückmeldungen ohne soziale Bewertung (durch die Notenskala) können hier Abhilfe schaffen (z.B. Gasztner 1978, Herber et al. 1978, Herber 1983, 1987, 1998d).

\section{Ad 1.1.8 Erfolgsmotivation (und Misserfolgsmotivation)}

Jeder Schüler/jede Schülerin ist (bereichspezifisch) mehr oder weniger erfolgsoder misserfolgsorientiert.

Wenn Schüler überwiegend erfolgsorientiert sind, sollten sie mittelschwierige (die Lösungswahrscheinlichkeit innerhalb der Population wäre etwa 50\%), mitunter auch relativ schwierige Herausforderungslagen (etwa 25\% Lösungswahrscheinlichkeit) durch entsprechende Aufgaben erleben können (Astleitner \& Herber 1993, Fuchs et al. 2001, Herber \& Vásárhelyi 2003).

Wenn sie misserfolgsmotiviert sind, sollten Sequenzen von Erarbeitungsaufgaben zur Verfügung stehen, die sie „sicher“ von Erfolg zu Erfolg führen (mindestens $70 \%$ Lösungswahrscheinlichkeit). Erst wenn sie genug Selbstvertrauen „getankt" haben, sollten sie aus sich heraus Möglichkeiten finden, auch anspruchsvolle, aber nicht zu schwierige Aufgaben zu wählen. Sie sollten gezielt Hilfe in Anspruch nehmen können - entweder durch Mitschüler (bei freigewählter Sozialform des Unterrichts), oder durch Lehrer- bzw. Computerunterstützung mittels bereit gestellter „Hilfeschleifen“, die man - selbst gewählt - in Anspruch nehmen kann (vgl. Herber 1983, Fuchs et al. 2001, Vásárhelyi 2004a,c, siehe auch unten Kap. 3). Je sachlicher und sparsamer verstärkende Leistungsrückmeldung kommt, desto mehr wird extrinsische (von außen kommende) Verstärkung zu quasi selbstgewollter, sachbezogener (intrinsischer) Motivation transformiert (vgl. das Prinzip der funktionellen Autonomie der Motive von Allport 1961/1970). Hoch leistungsmotivierte, erfolgsorientierte Personen sind eher an ihren eigenen Leistungen als an den Leistungen anderer Menschen orientiert. Es konnte empirisch gezeigt werden, dass normative Leistungsrückmeldungen (z.B. über soziale oder fremdgesetzte Bezugsnormen sensu Heckhausen 1974) keinen Einfluss auf die Leistung hoch- und erfolgsorientierter Vpn haben. Individuelles Feedback steigert hingegen deutlich deren Leistungen, insbesondere wenn sie eine Sache noch nicht völlig meistern oder in ihrem Leistungsniveau abzusinken drohen (z.B. Langens \& Schüler 2003). Man kann hoch erfolgsmotivierte Personen geradezu demotivieren, wenn man ihnen von außen vorschreibt, welche Aufgaben sie wie zu erledigen haben. Menschen 
"herber" — 2006/6/26 — 10:27 — page 23 — \#23

Kompetenzstreben und Kompetenzerwerb: Funktionale didaktische Fördermöglichkeiten. . 23

mit einem starken erfolgsorientierten Leistungsmotiv haben hohe selbstregulatorische Kompetenzen und reagieren mit „Reaktanz“ (Brehm 1966) bzw. „Ausdem-Feld-Gehen" (Lewin 1982), wenn das Ausleben dieser Kompetenzen durch fremdgesteuerte normative Regulative be- oder verhindert wird. Auch das spricht für I. D. und Individualisierung im Unterricht.

\section{Ad 1.2 Kompetenzerwerb im kognitiven Bereich}

Die kognitiven Lernvoraussetzungen sicher zu stellen, ist der wesentliche einleitende Schritt im Modell der I. D. nach Herber (1983). Bevor ein neuer Sachbereich - problemorientiert - erarbeitet werden kann, sind die notwendigen Wissensvoraussetzungen (Fakten, Begriffe, Algorithmen, etc.) und für analogiebildendes Problemlösen relevante kognitive Strukturen und operative Strategien in gezielter Wiederholung zu erheben - am besten in Form eines formativen Tests). Auf dieser Basis können Aufgabenstellungen des neuen Problemfeldes in individualisierter Form (Vásárhelyi 2004a,c) oder wenigstens - nach Mängeltypen - differenziert (Herber 1998c,d) angeboten werden (vgl. dazu unten Kap. 3). Wichtig ist, dass Lernende, die gravierende „Lücken“ im fundamentalen Bereich haben, vorweg - mit Lehrer- oder Computerhilfe - diese Lücken schließen können, bevor sie in den neuen Problembereich „einsteigen“, während Schüler ohne fundamentale Lücken in ihren Lernvoraussetzungen sich direkt dem anstehenden Thema widmen können. Für potentiell misserfolgängstliche Problemlöser sollte dabei ein „sicherer Erarbeitungspfad“ bereit gestellt werden (beginnend, intermittierend verstärkend und endend mit relativ leicht zu erfüllenden Arbeitsaufträgen, während zwischendurch auch mittlere Herausforderungslagen angeboten werden, damit ausreichende Lösungsspannungen und der Anschluss an Leistungstüchtigere nach Möglichkeit erhalten bleiben). Erfolgsmotivierte Lernende dürfen ihr „Aufgabenmen̈̈“ weitgehend selbst zusammen stellen, sie können aber jederzeit - bei etwaiger Überforderung - zum didaktisch gesicherten Erarbeitungspfad zurückkehren. Im didaktischen „Idealfall“ können sie ständig zwischen alternativen, sachstrukturell gesicherten Zugangswegen wählen, diese kombinieren und eigenständig ergänzen (siehe unten Kap. 3).

Alle Schüler entscheiden selbst, inwieweit sie individuell bzw. in Partner- oder Gruppenarbeit die (selbst)gestellten Aufgaben bewältigen wollen.

Sachlogisch können gewisse Aufgabenmodule für alle vorgesehen werden („Kriteriumsleistung“), damit der (sachlogische) Aufbau eines entsprechenden Lösungsrationales prototypisch abgesichert werden kann (Herber \& Vásárhelyi 2002b, Vásárhelyi 2004b). 
Die hier implizit dargestellten Aussagen über kognitiv-emotionale Interaktionen leiten uns zur didaktischen Reflexion des nächsten Kapitels über:

\section{Ad 1.3 Wechselwirkungen von kognitiven und emotional-motivationalen Prozessen}

Nach der PSI-Theorie von Kuhl (2001) kommt es nicht so sehr darauf an, Lernen mit positiven Affekten zu verknüpfen (das würde u. U. zu flachem „trial and error"-Verhalten führen). Es kommt darauf an problemsensitiv zu werden, doch nicht gleich zu versagen, wenn sich eine Lösung nicht sofort einstellt. Der mit Problemsensitivierung eingehende negative Affekt motiviert analytisches, genaues Denken, um das Problem schärfer zu fassen, zu dekomponieren und nach entsprechenden Lösungen zu suchen (z.B. durch das vergleichende Analysieren alternativer, analoger, doch sachlogisch adäquater Lösungswege, zusätzlicher didaktischer Hilfen, etc. - siehe unten Kap. 3). Stellen sich auf diese Weise keine (oder unzureichende) Lösungsansätze ein, sollte man „abschalten“, mittels Extensionsgedächtnis die ganze weite „Landschaft“ des zur Verfügung stehenden Kontext- und Selbstwissens aktivieren, (akkomodierende) Umstrukturierungen des Problems versuchen und - mittels Analogiebildungen - neue Kombinationen bereits vorhandener Lösungsansätze suchen. In unserem Modell der I. D. (z.B. Herber \& Vásárhelyi 2002b) wird diese bei mittlerer Aktivierung erzielte optimale Umstrukturierungsleistung der emotional-kognitiven Funktionen in mehrfacher Hinsicht angestrebt ( $\Leftrightarrow$ der "herabregulierte" negative Affekt des analytischen Modus entspricht in seiner Intensität in etwa dem durch weitläufige Kontext- und Selbstanbindung „heraufregulierten“ positiven Affekt, wodurch beide Hirnhälften zusammengeschaltet werden und somit maximale Kapazitätsnutzung hergestellt werden kann, vgl. Kuhl 2001):

- Auf der Basis eines gesicherten Pfades von Lernschritten (zu dem man immer „zurückkehren“ kann) - oder besser: mehrerer äquivalenter "Sicherungspfade“ (sieh unten Kap. 3) - können selbstständig alternative Problem-/Lösungssequenzen aufgesucht, selbst konstruiert, in individueller Weise kombiniert, etc. werden. So werden selbstgesteuert die optimalen „mittleren“ Diskrepanzerlebnisse aktiviert, die Problem(um)strukturierungen am ehesten ermöglichen.

- Die Sozialform kann - spätestens ab der Sekundarstufe I - von den Schülern frei gewählt werden, so dass eine optimale Affektlage extrinsisch gestützt werden kann (falls sachbezogen-intrinsische Interessen entweder zu wenig vorhanden sind oder eine übermäßig fixierende Interessiertheit zu individueller Übererregung führt, die durch soziale Ablenkung „beruhigt“ werden kann). 
- Durch selbstgesteuerten Wechsel zwischen haptischem „Be-Greifen“ (geeignete Objekte können zerlegt, zusammengebaut, selbst manuell hergestellt werden), „paper and pencil“-fundierten ikonischen und symbolischen Repräsentationen und der gezielten Nutzung von entsprechenden ComputerAnimationen) kann eine ideale Strukturierung mittels Re-Kombination bekannter Lösungsstrategien bei selbstgesteuerter mittlerer Affekt-/Aktivierungslage erreicht werden (vgl. Lewalter 1997, siehe auch unten Kap. 3).

- Die für mittlere Aktivierung/Erregung und optimale kognitive Umstrukturierungsmöglichkeiten ideale Zusammenschaltung des analytischen und des ganzheitlichen Modus erfordert weitgehende Selbststeuerungsmöglichkeiten in einem grundsätzlich idiosynkratisch zu verstehenden Motivations- und Lernprozess. Sachlogisch notwendige Struktur- und Funktionsvorgaben (prototypische Kriteriumsleistungen) sollten nur im unbedingt erforderlichen Ausmaß die emotional-kognitive Informationsverarbeitung des Lernenden lenken. So weit als möglich sollten - bei Sicherung eines fundametalen Erarbeitungsmodus (auf der man im Sinne fundamentaler didaktischer Hilfen immer zurückgreifen kann) - die individuellen Stimmungs-, Temperamentsund Motivationslagen sich im Wechselspiel zwischen sachlichen und sozialen Möglichkeiten (und Notwendigkeiten) „frei“ entfalten können. Didaktische Interventionen sollten mehr der Schadens-(Fehler-)Prävention dienen als dem willkürlichen Eingreifen „von außen“ Vorschub leisten. Dem Prinzip der „minimalen Lernhilfen" (Zech 1998) kann lern- und motivationstheoretisch oberste didaktische Priorität zuerkannt werden.

\section{Prototypische Aufgabenstellungen zur Förderung vom Kompetenzstreben und Kompetenzerwerb im Mathematikunterricht - Problemlösen durch geometrische Repräsentation}

Dieses Beispiel soll zeigen, wie mit parallel angebotenen didaktischen $\mathrm{Zu}$ gangswegen verschiedene Kompetenzen bzw. Kompetenzmotivationen angesprochen werden können, indem Lernende der Sekundarstufe II ihre eigenen Kombinationen von Aufgaben und damit - sachstrukturell abgesichert - individuelle Lösungswege generieren können. 
Die Lerneinheit ist in elektronischer Form im Internet erreichbar (Vásárhelyi 2004d). Die Erarbeitung der Arbeitsblätter kann durch traditionelle Arbeitsblätter gelenkt und protokolliert werden. Dementsprechend kann man sie z.B. als Aufgabenserie für selbständiges Lernen oder als Hilfestellung bzw. als Ergebniskontrolle für den Problemlöseprozess einsetzen. Darüber hinaus aber werden die mittels Arbeitsblatt gewonnenen „festen Bilder" durch die damit verbundenen Computeranimationen wieder „beweglich“ gemacht, was u.a. das räumliche Vorstellungsvermögen fördert (Lewalter 1997) und insgesamt - im Sinne des PhiPhänomens von Wertheimer (1912) - einen emergenten, dynamischen Umgang mit den geometrischen "Veranschaulichungen" arithmetischer Problemstellungen ermöglicht. So kann jeder Lernende die benötigten Lernschritte nach seiner Kompetenz(motivation) jeweils verkleinern bzw. vergrößern.

Zur Initiierung von Problemlöseprozessen wird ein Problem ins Zentrum gestellt und die anderen Aufgaben werden als Hilfestellungen und Aktivierung der Lernvoraussetzungen bzw. als Erweiterung betrachtet. Im Zentrum steht das Problem [Aufgabe d)], wie man die Summe von Quadratzahlen bestimmen kann. Dazu werden 4 Lösungswege ( $\mathrm{Ld}$ ) angeboten mit Querverbindungen (Hyperlinks) zu den methodischen und inhaltlichen Lernvoraussetzungen [Aufgaben a) - c) mit jeweils mehreren Lösungswegen ( $\mathrm{La}-\mathrm{Lc}$ )], zu den Hilfestellungen $(\mathrm{Ha}-\mathrm{Hd}$ ), Kontrollstellen ( $\mathrm{Ka}$ - $\mathrm{Kd}$ ) und zur additionalen Anwendung der Methode bzw. des Ergebnisses [Aufgabe ${ }^{*}$ ) mit $\mathrm{He}$, Le und $\mathrm{Ke}$ ]. ${ }^{*}$ bedeutet im Sinne der Inneren Differenzierung die Additumsaufgabe bzw. weiterführende Bemerkungen.

\subsection{Aufgabenstellungen zum Thema positive ganze Zahlen}

Aufgabe a) Bestimmen Sie die Summe der ersten $n$ positiven ganzen Zahlen:

$$
1+2+3+\ldots+n=? \quad \mathrm{Ha} \mathrm{La}
$$

Aufgabe b) Bestimmen Sie die Summe der ersten $n$ positiven geraden Zahlen:

$$
2+4+6+\ldots+2 n=? \quad \mathrm{Hb} \mathrm{Lb} \mathrm{Kb}
$$

Aufgabe c) Bestimmen Sie die Summe der ersten $n$ positiven ungeraden Zahlen:

$$
1+3+5+\ldots+2 n-1=? \quad \mathrm{Hc} \mathrm{Lc} \mathrm{Kc}
$$


Aufgabe d) Bestimmen Sie die Summe $S(n)$ der Quadrate der ersten $n$ positiven ganzen Zahlen:

$$
S(n)=1^{2}+2^{2}+3^{2}+\ldots+n^{2}=? \quad \mathrm{Hd} \mathrm{Ld} \mathrm{Kd}
$$

*Aufgabe e) Bestimmen Sie die folgende Summe:

$$
1 \cdot 2+2 \cdot 3+3 \cdot 4+\ldots+(n-1) \cdot n=? \quad \mathrm{He} \mathrm{Le} \mathrm{Ke}
$$

\subsection{Hilfestellungen zu den einzelnen Aufgaben}

Bei den direkten Hilfestellungen zu den einzelnen Aufgaben ist es wichtig, dass die anregende Frage oder die kurze Information dem Lernenden das Problemlösen nicht abnehmen darf.

$\mathrm{Ha}$ Experimentieren Sie mit konkreten Zahlen.

$\mathrm{Hb}$ Die geraden Zahlen sind das Doppelte von ganzen Zahlen. Die Aufgabe b) lässt sich auch nach der 2. Lösungsmethode der Aufgabe a) lösen.

Hc Sie können die Ergebnisse und die Lösungsmethode der Aufgaben a) bzw. b) verwenden.

Hd Die vorherigen Methoden ermöglichen einige algebraische Lösungen.

$\mathrm{He}$ Wir betrachten die Pyramide, die wir bei der Lösung 2 der Aufgabe d) verwendet haben. Davon lassen wir jetzt eine vertikale Schicht weg. Nachdem wir das Ergebnis der Aufgabe d) kennen, genügt es, sich auf die ganzen Würfel zu konzentrieren.

\subsection{Kontrollstellen zu den einzelnen Aufgaben}

Die Ergebnisse sind durch Hyperlinks an den Kontrollstellen $\mathrm{Ka}-\mathrm{Ke}$ direkt erreichbar, falls der Lernende bislang selbständig gearbeitet hat und sich danach kontrollieren will.

$$
\begin{aligned}
& 1+2+3+\ldots+n=\frac{n(n+1)}{2} \\
& 2+4+6+\ldots+2 n=n(n+1) \\
& 1+3+5+\ldots+(2 n-1)=n^{2}
\end{aligned}
$$

$$
S(n)=1^{2}+2^{2}+3^{2}+\ldots+n^{2}=\frac{n(n+1)(2 n+1)}{6}
$$




$$
1 \cdot 2+2 \cdot 3+3 \cdot 4+\ldots+(n-1) \cdot n=\frac{n(n+1)(2 n+1)}{3}
$$

\subsection{Lösungswege zu den einzelnen Aufgaben}

Ein arithmetischer Lösungsweg wird konsequent betreut und zusätzlich werden mehrere Lösungswege durch geometrische Interpretation angeboten. Es ist möglich während der didaktisch angeleiteten Erarbeitung zum Problemlösen zurückzukehren, auch wenn der Lernende statt der selbständigen Arbeit das „Lesen“ wählt. Die „Animationen“ sind interaktive Arbeitsblätter, wo die Lernenden nach Bedarf Hilfe holen können, oft kann die Figur (ein Bildschirmausschnitt aus der Animation) schon genügende Anregung geben um die Arbeit selbständig fortsetzen zu können (z.B. das Bild bei Animation 2). Natürlich kann der Rückgriff auf haptische Erarbeitungsformen für manche Schüler das Problemerfassen und -lösen erleichtern. Unsere Kommentare beziehen sich vor allem auf den Einsatz der elektronischen Arbeitsblätter, so dass in diesem Zusammenhang nicht alle möglichen (eventuell von den Schülern erfundenen) Lösungsvarianten diskutiert werden können.

\section{La Ad Aufgabe a)}

Lösung 1 der Aufgabe a): Wir lösen den konkreten Fall $n=6$ zuerst so, wie Gauss die Aufgabe als Kind gelöst hat: Er hat die Zahlen in die erste Zeile steigend, in die zweite Zeile fallend aufgeschrieben und die Zahlen in den Spalten addiert.

Dabei hat er gemerkt, dass

- die Summe bei jeder Spalte mit der Summe der ersten und der letzten Zahl gleich ist $(7=1+6)$

- die Summe der Spaltensummen $(6 \cdot(1+6))$ das Doppelte der gesuchten Summe beträgt:

$$
\begin{aligned}
& 1+2+3+4+5+6 \\
& \frac{6+5+4+3+2+1}{7+7+7+7+7+7} \\
& 1+2+3+4+5+6=\frac{6 \cdot 7}{2}
\end{aligned}
$$

Lösung 2 der Aufgabe a): Wir bauen auf dem Zahlenstrahl eine „Treppe“ so auf, dass die Höhe des Streifens zwischen $k-1$ und $k$ eben $k$ ist (der untere Teil in der Figur). 


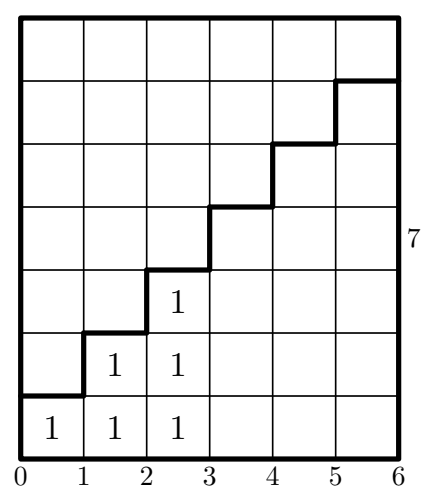

Der Flächeninhalt der „Treppe“ ist eben die Summe der ersten 6 positiven ganzen Zahlen. In der Figur kann man sehen, dass ein Rechteck mit den Seitenlängen 6 und $(6+1)$ zwei solche „Treppen“ enthält. So stimmt der Flächeninhalt der „Treppe“ mit der Hälfte des Flächeninhalts des Rechtecks überein:

$$
1+2+3+4+5+6=\frac{6 \cdot 7}{2}
$$

Beide Verfahren lassen sich für jede beliebige positive ganze Zahl anwenden. Folglich ist die Summe der ersten $n$ positiven ganzen Zahlen:

$$
1+2+3+\ldots+n=\frac{n \cdot(n+1)}{2} .
$$

\section{Lb Ad Aufgabe b)}

Lösung 1 der Aufgabe b): Aus dem Ergebnis der Aufgabe a) erhalten wir

$$
2 \cdot(1+2+3+\ldots+n)=2 \cdot \frac{n \cdot(n+1)}{2}=n \cdot(n+1) .
$$

Lösung 2 der Aufgabe b): Wir betrachten ein Quadrat der Seitenlänge $n$. Wir halbieren die Einheitsquadrate durch Diagonalen und betrachten diese kleinen Dreiecke als „neue Flächeninhaltseinheiten“. Wir wählen die kleinen Dreiecke unter der Diagonalen des Quadrats zusammen mit den kleinen Dreiecken der oberen Hälfte des Quadrats, deren Hypotenuse auf der Diagonalen liegt. Der Flächeninhalt der ausgewählten Dreiecke ist eben die Summe der geraden Zahlen (zeilenweise abgezählt). Aus den $2 n^{2}$ Dreiecken haben wir die in der unteren Hälfte enthaltenen $n^{2}$ und die entlang der Diagonale liegenden n Stück ausgewählt:

$$
2+4+6+\ldots+2 n=n^{2}+n=n(n+1) .
$$




$$
\text { "herber" — 2006/6/26 — 10:27 — page } 30-\# 30
$$

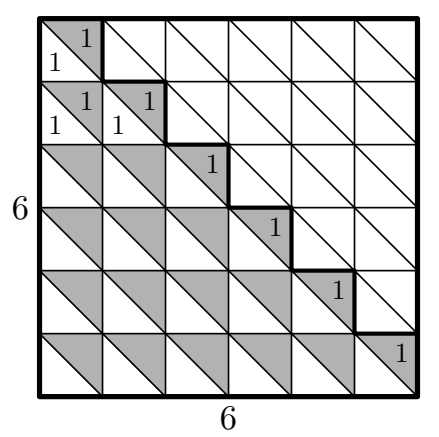

\section{Lc Ad Aufgabe c)}

Lösung 1 der Aufgabe c): Die Ergebnisse der Aufgaben a) und b) verwenden wir für $2 n$ :

$$
\begin{aligned}
& 1+3+5+\ldots+(2 n-1)= \\
& \quad=(1+2+3+\cdots+2 n)-(2+4+6+\cdots+2 n) \\
& =n(2 n+1)-n(n+1)=n^{2} .
\end{aligned}
$$

Ein anderer Weg ist nach der Definition der ungeraden Zahlen $(2 k-1)$ möglich:

$$
\begin{aligned}
1+3 & +5+\ldots+(2 n-1)= \\
& =(2-1)+(4-1)+(6-1)+\ldots+(2 n-1) \\
& =(2+4+6+\ldots+2 n)-n=n(n+1)-n=n^{2} .
\end{aligned}
$$

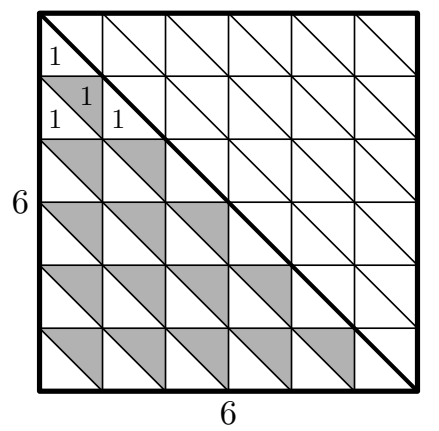

Lösung 2 der Aufgabe c): Wir kehren zur Lösung 2 der Aufgabe b) zurück. Die Einheitsquadrate des Quadrats der Seitenlänge $\mathrm{n}$ werden wieder in 2 kongruente Dreiecke zerlegt, und diese werden als Flächeninhaltseinheit betrachtet. 
Die Dreiecksarithmetik betrifft jetzt die Dreiecke unter der Diagonalen des großen Quadrats, wir addieren diese zeilenweise und erhalten die Summe der ungeraden Zahlen:

$$
1+3+5+\ldots+(2 n-1)=n^{2}
$$

*Bemerkung: Wir haben bewiesen, dass die Summe eine Quadratzahl ist.

Lösung 3 der Aufgabe c): Die Lösungsidee stammt aus der Vermutung, dass die Summe eine Quadratzahl ist.

Diese Vermutung kann man aus einer Zeichnung erhalten:

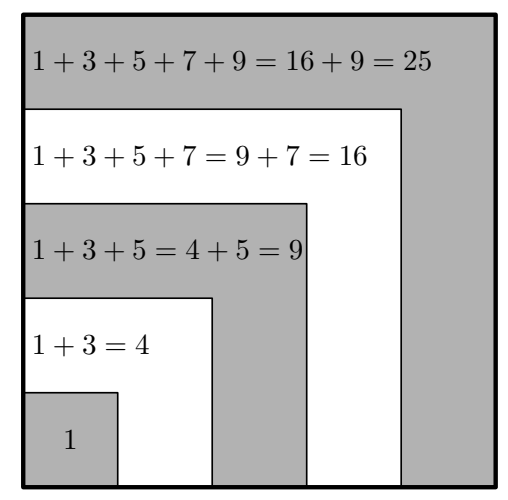

Abbildung 2. Bildschirmausschnitt aus der Animation 1

Wir zeichnen ein Einheitsquadrat und ergänzen dieses um ein Quadrat der Seitenlänge 2, d.h. dass wir die 4 Quadrate in der Form $1+3$ gruppieren. Das Quadrat der Seitenlänge 2 lässt sich zu einem Quadrat der Seitenlänge 3 ergänzen, usw. Es ist zu beweisen, dass sich der Prozess von $(n-1) \cdot(n-1)$ auf $n \cdot n$ fortsetzen lässt. Nach Induktionsannahme ist die Summe der ersten $(n-1)$ positiven ungeraden Zahlen eine Quadratzahl:

$$
1+3+5+\ldots+2(n-1)-1=(n-1)^{2}
$$

$\mathrm{Zu}$ beiden Seiten addieren wir $(2 n-1)$, dadurch bekommen wir auf der linken Seite die Summe der ersten $n$ ungeraden positiven Zahlen,

$$
1+3+5+\ldots+2(n-1)-1+2 n-1=(n-1)^{2}+2 n-1=n^{2} .
$$




\section{Ld Ad Aufgabe d)}

Lösung 1 der Aufgabe d): Wir beginnen mit der Summe der Quadrate der ersten 4 positiven ganzen Zahlen. Wie in der Figur zu sehen ist, bauen wir aus den Quadraten der Seitenlänge 1, 2, 3 und 4 einen „Turm“. Wir lassen ein Einheitsquadrat neben dem „Turm" frei und zeichnen das Spiegelbild des „Turms“. Wir überdecken die zwei „Türme“ (gemeinsam) durch ein Rechteck der Seitenlänge $(4+1+4)$ und $(1+2+3+4)$.

Der Flächeninhalt des Rechtecks ist $F=(2 \cdot 4+1)(1+2+3+4) . F$ lässt sich auch anders bestimmen: Den mittleren Teil zerlegen wir in Streifen der Breite 1. Dadurch erhalten wir 4 Streifen der Länge 1, 3 Streifen der Länge 3, 2 Streifen der Länge 5 und einen Streifen der Länge 7 .

Den Flächeninhalt der Streifen addieren wir mit der folgenden Gruppierung:

$$
(1+3+5+7)+(1+3+5)+(1+3)+(1) .
$$

Aus der Aufgabe c) folgt $1+3+\ldots+2 k-1=k^{2}$, so ist der Flächeninhalt des mittleren Teils eben $S(4)$, und der Flächeninhalt des Rechtecks ist $F=3 \cdot S(4)$.

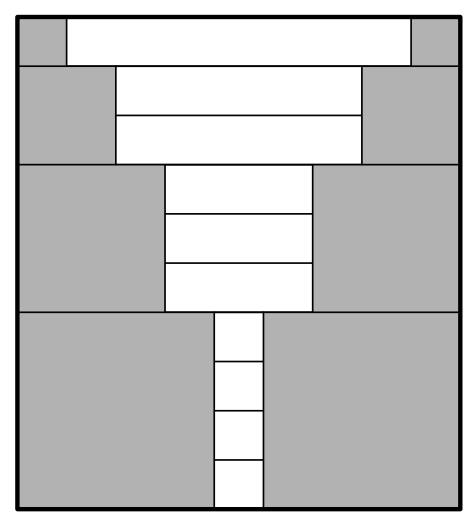

Abbildung 3. Bildschirmausschnitt aus der Animation 2

Aus dem Vergleich der zwei Berechnungen von $F$ erhalten wir

$$
S(4)=\frac{4 \cdot(4+1) \cdot(2 \cdot 4+1)}{6} .
$$

Der obige Prozess lässt sich verallgemeinern: Aus den Seitenlängen wird der Flächeninhalt des Rechtecks errechnet:

$$
F=(2 n+1)(1+2+3+\ldots+n)=(2 n+1) \cdot \frac{n(n+1)}{2} .
$$




$$
\text { "herber" — 2006/6/26 — 10:27 — page } 33 \text { — \#33 }
$$

Kompetenzstreben und Kompetenzerwerb: Funktionale didaktische Fördermöglichkeiten. . 33

Aus der Summe der Teilflächeninhalte folgt $T=3 \cdot S(n)$, und daraus erhalten wir:

$$
S(n)=\frac{n \cdot(n+1) \cdot(2 n+1)}{6} .
$$

Lösung 2 der Aufgabe d): Die Summe der Quadratzahlen suchen wir als Volumen einer aus Einheitswürfeln zusammengesetzten Pyramide. In der Figur ist der Fall $n=4$ zu sehen: aus 1, 4, 9 und 16 Einheitswürfeln wurde jeweils eine quadratische Säule der Höhe 1 gebaut und diese Schichten haben wir aufeinander gestellt.

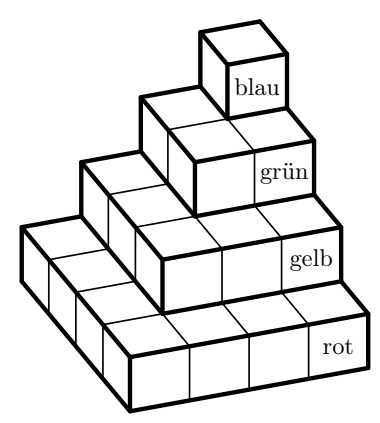

Abbildung 4. Bildschirmausschnitt aus der Animation 3

Der so erhaltene Turm wurde zu einer quadratischen Pyramide ergänzt, indem die entsprechenden Anzahlen von Dritteln bzw. Hälften des Einheitswürfels hinzugefügt wurden:

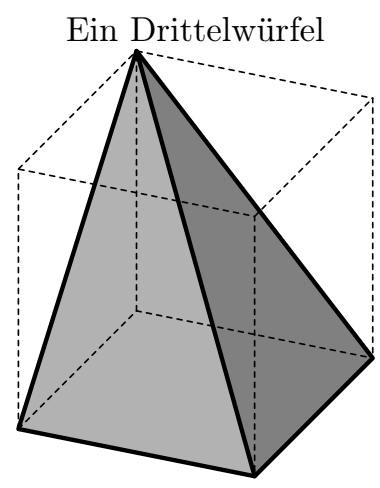

Abbildung 5. Bildschirmausschnitt aus der Animation 4 
Auf den oben befindlichen einzelnen Würfel $\left(\right.$ blau $\left.^{2}\right)$ wurde ein Drittelwürfel (kleine Pyramide) gestellt, an zwei Seitenflächen je ein Halbwürfel und zu der gemeinsamen Kante von diesen wieder ein Drittelwürfel. Dadurch steht über der zweiten (grünen) Schicht ein Drittel des Würfels der Seitenlänge 2. Die zweite Schicht ergänzen wir durch 4 Halbwürfel und einen Drittelwürfel und erhalten so über der dritten (gelben) Schicht einen Drittelwürfel der Länge 3. So setzen wir mit den nächsten Schichten (gelb, rot, ...) fort. Unter der letzten Schicht kann man eine weitere Schicht bauen, usw.

Die folgende Tabelle enthält die Verteilung der Bauelemente nach Schichten.

\begin{tabular}{|c|c|c|c|}
\hline Schicht & Ganze & Halbe & Drittel \\
\hline 1. & 0 & 0 & 1 \\
\hline 2. & 1 & $2 \cdot 1$ & 1 \\
\hline 3. & $2^{2}$ & $2 \cdot 2$ & 1 \\
\hline 4. & $3^{2}$ & $2 \cdot 3$ & 1 \\
\hline$\ldots$ & $\ldots$ & $\ldots$ & $\ldots$ \\
\hline$(n+1)$. & $n^{2}$ & $2 \cdot n$ & 1 \\
\hline
\end{tabular}

Wenn wir $n$ Schichten aus Einheitswürfeln haben, enthält ein Drittelwürfel der Seitenlänge $(n+1)$ alle ganzen Einheitswürfel, deren Gesamtvolumen wir suchen. Der Inhalt des Drittelwürfels kann aber auch mit Hilfe der Tabelle bestimmt werden: Das Gesamtvolumen der ganzen Würfel entspricht eben der gesuchten Summe $S(n)$, die Anzahl der Halbwürfel ist das Doppelte der Summe der ersten $n$ positiven ganzen Zahlen, die Anzahl der Drittelwürfel ist $n+1$. Daraus folgt:

$$
\begin{gathered}
V=S(n)+\frac{n(n+1)}{2}+\frac{n+1}{3}=\frac{(n+1)^{3}}{3}, \\
S(n)=\frac{(n+1)^{3}}{3}-\frac{n(n+1)}{2}-\frac{n+1}{3}=\frac{n(n+1)(2 n+1)}{6} .
\end{gathered}
$$

Lösung 3 der Aufgabe d): Wir bestimmen die Summe $(1-1)^{3}+(2-1)^{3}+$ $\ldots+(n-1)^{3}$ mit Hilfe der Gleichung $(a-b)^{3}=a^{3}-3 a^{2} b+3 a b^{2}-b^{3}$.

${ }^{2}$ Die Farben beziehen sich auf die Körpermodelle, Animationen, Abbildungen (Bildschirmausschnitte), die in der realen Lernsituation verwendet wurden bzw. zu verwenden sind (siehe z.B. Vásárhelyi 2003). 


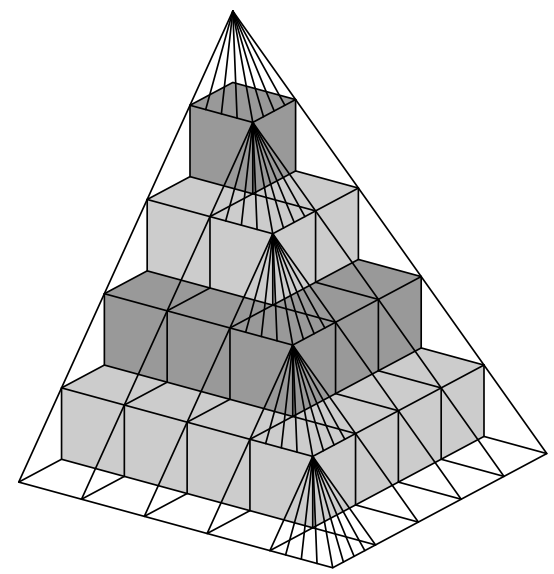

Abbildung 6. Bildschirmausschnitt aus der Animation 5

\begin{tabular}{|rlllllllll|}
\hline $0^{3}$ & $=$ & $(1-1)^{3}$ & $=$ & $1^{3}$ & - & $3 \cdot 1$ & + & $3 \cdot 1^{2}$ & -1 \\
\hline $1^{3}$ & $=$ & $(2-1)^{3}$ & $=$ & $2^{3}$ & - & $3 \cdot 2$ & + & $3 \cdot 2^{2}$ & -1 \\
\hline $2^{3}$ & $=$ & $(3-1)^{3}$ & $=$ & $3^{3}$ & - & $3 \cdot 3$ & + & $3 \cdot 3^{2}$ & -1 \\
\hline $3^{3}$ & $=$ & $(4-1)^{3}$ & $=$ & $4^{3}$ & - & $3 \cdot 4$ & + & $3 \cdot 4^{2}$ & -1 \\
\hline$\ldots$ & $\ldots$ & $\ldots$ & $\ldots$ & 7 & $\ldots$ & & & \\
\hline$(n-2)^{3}$ & $=$ & $((n-1)-1)^{3}$ & $=$ & $(n-1)^{3}$ & $-3 \cdot(n-1)$ & $+3 \cdot(n-1)^{2}$ & -1 \\
\hline$(n-1)^{3}$ & $=$ & $(n-1)^{3}$ & & $n^{3}$ & - & $3 \cdot n$ & + & $3 \cdot n^{2}$ & -1 \\
\hline
\end{tabular}

Wir addieren alle diese Gleichungen und lassen die Terme weg, die auf beiden Seiten vorkommen.

$$
0=n^{3}-3 \cdot(1+2+3+\ldots+n)+3 \cdot\left(1^{2}+2^{2}+3^{2}+\ldots+n^{2}\right)-n .
$$

Daraus folgt durch Äquivalenzumformungen

$$
S(n)=1^{2}+2^{2}+3^{2}+\ldots+n^{2}=\frac{n-n^{3}}{3}+\frac{n(n+1)}{2}=\frac{n(n+1)(2 n+1)}{6} .
$$

*Bemerkung: Mit dieser Methode kann man auch die Summe der dritten Potenzen bestimmen, denn die Summe der Quadrate ist schon bekannt. So lässt sich die Sammlung der Formeln erweitern: Mit Hilfe der dritten Potenzen die Summe von vierten Potenzen, usw. ... 
Lösung 4 der Aufgabe d): In einer quadratischen Tabelle schreiben wir in jede Zeile die positiven ganzen Zahlen von 1 bis $n$ auf. Die Summe dieser Zahlenfolgen wird auf zwei Wegen bestimmt.

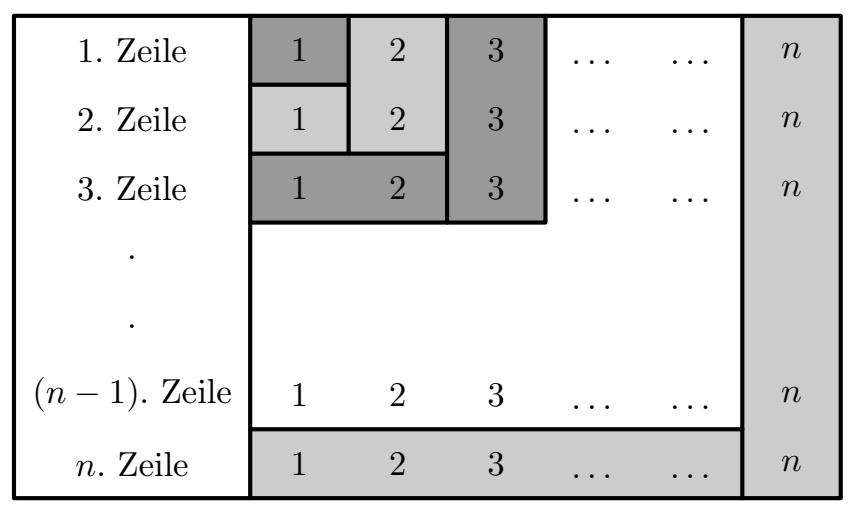

Zuerst addieren wir die Zahlen zeilenweise, das ist $n$-mal die Summe der positiven ganzen Zahlen von 1 bis $n$ :

$$
n(1+2+3+\ldots+n)=\frac{n^{2}(n+1)}{2} .
$$

Zweitens addieren wir die Zahlen in den $L$-förmigen Streifen der Breite 1 und dann addieren wir diese Teilsummen. Die Summe der Zahlen, die in dem $L$-Streifen liegen, der „oben“ mit $k$ beginnt, ist

$$
\frac{(k-1) k}{2}+k^{2}=\frac{3}{2} k^{2}-\frac{1}{2} k
$$

Jetzt lassen wir $k$ von 1 bis $n$ laufen und addieren diese Teilsummen:

$$
\frac{3}{2} \cdot\left(1^{2}+2^{2}+3^{2}+\ldots+n^{2}\right)-\frac{1}{2} \cdot(1+2+3+\ldots+n)
$$

Aus dem Vergleich von beiden Summen ergibt sich:

$$
\begin{aligned}
S(n) & =\left(1^{2}+2^{2}+3^{2}+\ldots+n^{2}\right)= \\
& =\frac{2}{3}\left(\frac{n^{2}(n+1)}{2}+\frac{1}{2} \cdot \frac{n(n+1)}{2}\right)=\frac{n(n+1)(2 n+1)}{6} .
\end{aligned}
$$

\section{Le Ad Aufgabe ${ }^{*} e$ )}

Wir betrachten die Pyramide, die wir bei der 2. Lösung der Aufgabe d) verwendet haben. Davon lassen wir jetzt eine vertikale Schicht weg. Die zurückgebliebenen Bauelemente tragen wir in die Tabelle ein: 
$\underline{\text { Kompetenzstreben und Kompetenzerwerb: Funktionale didaktische Fördermöglichkeiten. . . } 37}$

\begin{tabular}{|c|c|c|c|}
\hline Schicht & $\begin{array}{c}\text { Ganze } \\
\text { Pyramide - abge- } \\
\text { schnitten = bleibt }\end{array}$ & $\begin{array}{c}\text { Halbe } \\
\text { Pyramide - abge- } \\
\text { schnitten = bleibt }\end{array}$ & $\begin{array}{c}\text { Drittel } \\
\text { Pyramide - abge- } \\
\text { schnitten = bleibt }\end{array}$ \\
\hline 1. & $0-0=0$ & $0-0=0$ & $1-1=0$ \\
\hline 2. & $1-1=0$ & $2 \cdot 1-1=1$ & 1 \\
\hline 3. & $2^{2}-2=2$ & $2 \cdot 2-1=3$ & 1 \\
\hline 4. & $3^{2}-3=6$ & $2 \cdot 3-1=5$ & 1 \\
\hline$\ldots$ & $\ldots$ & $\ldots$ & 1 \\
\hline$(n+1)$. & $n^{2}-n$ & $2 \cdot n-1$ & $\ldots$ \\
\hline
\end{tabular}

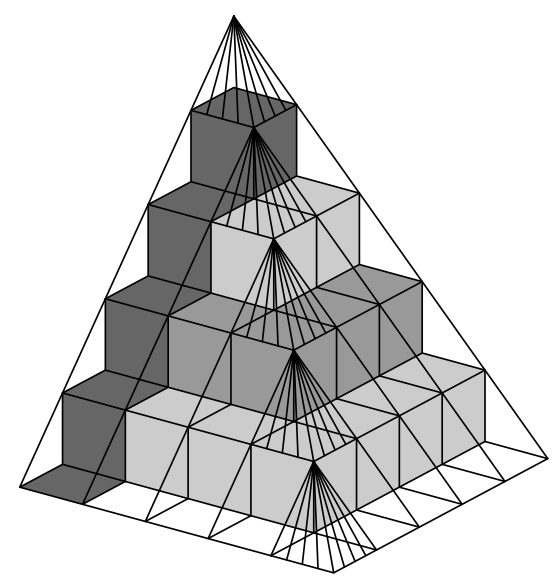

Die Anzahl der ganzen Würfel:

$$
\begin{aligned}
1 \cdot 2 & +2 \cdot 3+3 \cdot 4+\ldots+(n-1) \cdot n= \\
& =1-1+2^{2}-2+3^{2}-3+\ldots+n^{2}-n= \\
& =\frac{n(n+1)(2 n+1)}{6}-\frac{n \cdot(n+1)}{2} .
\end{aligned}
$$

\section{Schlussbemerkungen}

Der diagnostische Ausgangspunkt für Maßnahmen der Inneren Differenzierung und Individualisierung ist die Tatsache, dass eine Lernumgebung, die für manche Individuen (oder „Lerntypen“) förderlich ist, nicht notwendigerweise auch 
"herber" — 2006/6/26 — 10:27 — page 38 — \#38

für andere Individuen (Lerntypen) passt - oder gerade deswegen nicht förderlich, sondern sogar hinderlich ist (vgl. Herber 1983). Alle in obigen Zusammenhängen diskutierten, aus anerkannten Theorien abgeleiteten Handlungsempfehlungen müssen auf die im Grunde immer idiosynkratischen Kompetenzen und Kompetenzbestrebungen abgestimmt werden - am besten von den agierenden Individuen selbst. So sehr es im Allgemeinen stimmt, dass herausfordernde (mittelschwere) Aufgaben am ehesten die Ausbildung von Kompetenzen und Kompetenzstreben (im Sinne intrinsischer Motivation) fördern, so brauchen hochängstliche Schüler doch sehr viel mehr Erfolgserlebnisse und soziale (extrinsische) Stützung (Lob, Hilfe, Bestätigung, auf dem richtigen Weg zu sein) als erfolgszuversichtliche Lernende, die sehr viel rascher "medias in res" gehen wollen, um ihre Kompetenzen zu erproben und so ihr Selbstkonzept und Selbstwertgefühl zu bestätigen. Je weniger diesen von außen geholfen wird, desto mehr stärken sie ihr Kompetenzerleben mittels internaler Attribution (vgl. Dresel 2004, Aronson et al. 2004).

Den (illusionären) Anspruch aufzugeben, mit ein- und derselben didaktischen, gut vorbereiteten Maßnahme allen Schülern bestmöglich helfen und damit alle Lernaktivitäten kontrollieren zu können, fällt vielen Lehrern nicht leicht (Herber 1983). Das heißt auch, dass Lehrern nicht die ganze Verantwortung für das Lernergebnis der Schüler aufgehalst werden darf: Je mehr sie sich für den Lernfortschritt ihrer Schüler verantwortlich fühlen, desto direktiver und kritischer begleiten sie den Lernprozess der ihnen Anvertrauten, desto mehr versuchen sie, diese auf den (einzigen) „richtigen“ Weg hin zu manipulieren (z.B. Deci et al. 1982).

„Schüler sind soziale Wesen, keine Automaten, die man auf Knopfdruck mit Wissen füttern kann. Der Lehrer kann nur helfen, indem er versucht, auf diese je besonderen Wesen einzugehen, vorsichtig Hilfen im Sinne von Hypothesen anzubieten und ständig rückzukoppeln, ob seine ,Hilfe' hilfreich war oder nicht. ... Lehrer und Schüler müssen lernen, dass Lehrerhilfen nützen, aber auch behindern können. Keinesfalls aber sollte sich der Schüler alleingelassen fühlen. Er muss spüren (und oft genug gesagt bekommen), dass der Lehrer sein Bestes gibt, um ihm zu helfen. Dann wird er auch sein Bestes geben ... Ich möchte schließen ... mit der Bitte, über Idealismus und Perfektionswahn in der Schule - oft genug mit Bildung verwechselt - die realen Befriedigungsmöglichkeiten von Lehrern und Schülern nicht zu zerstören, wie dies durch narzisstisches Unsterblichkeitsstreben einzelner Machtträger auf allen Ebenen immer wieder geschieht." (Herber 1983, 117f.) 


$$
\text { "herber" — 2006/6/26 — 10:27 — page 39 — \#39 }
$$

Kompetenzstreben und Kompetenzerwerb: Funktionale didaktische Fördermöglichkeiten. . 39

\section{Literatur}

[1] J. Abel und C. Tarnai (Hg.), Pädagogisch-psychologische Interessenforschung in Studium und Beruf, Waxmann, Münster, 1998.

[2] A. Adler, Menschenkenntnis, Fischer, Frankfurt, 1927, Nachdruck 1966.

[3] M. Ainley, K. Hillman and S. Hidi, Gender and interest processes in response to literary texts: situational and individual interest, Learning and Instruction 12 (2002), 411-428.

[4] M. K. Alderman, Motivation for achievement. Possibilities for teaching and learning, Erlbaum, Mahwah, 1999.

[5] G. Allport, Gestalt und Wachstum in der Persönlichkeit, Hain, Meisenheim, 1970, (amerikanische Erstausgabe: Pattern and growth in personality, Holt, Rinehart \& Winston, New York, 1961).

[6] G. Ambrus, Üben in der Planung des Mathematikunterrichts, Unveröff., Salzburg, 2003, Dissertation.

[7] E. Aronson, T. D. Wilson und R. M. Akert, Sozialpsychologie, Pearson, München, 2004.

[8] P. Ashton and R. Webb, Making a difference: Teachers' sense of efficacy and student achievement, Longman, New York, 1986.

[9] P. Ashton, Motivation and teachers' sense of efficacy, in: Research on motivation in education, Vol. 2, The classroom milieu, (C. Ames and R. Ames, eds.), Academic Press, Orlando, 1985, 141-174.

[10] H. Astleitner und H.-J. Herber, Rechnersimulation von Auswirkungen unterschiedlicher Erfolgswahrscheinlichkeiten auf motivationale Prozesse, Humankybernetik 34 (1993), 78-88.

[11] H. Astleitner, Kritisches Denken - Basisqualifikationen für Lehrer und Ausbilder, Studien-Verlag, Innsbruck, 1998.

[12] H. Astleitner, Web-based instruction and learning, in: Towards the virtual university. International online learning perspectives, (N. Nistor, S. English and S. Wheeler, eds.), Information Age Publishing, Greenwich, 2003, 37-63.

[13] H. Astleitner, R. Brünken and S. Zander, Können Schüler und Lehrer kritisch denken?, Salzburger Beiträge zur Erziehungswissenschaft 6, Heft 2 (2002), 51-61.

[14] J. W. Atkinson and D. Birch, The dynamics of action, Wiley, New York, 1970.

[15] J. W. Atkinson and D. Birch, The dynamics of achievement-oriented activity, in: Motivation and achievement, (J. W. Atkinson and J. O. Raynor, eds.), Winston, Washington, 1974, 271-325.

[16] J. W. Atkinson and N. T. Feather (eds.), A theory of achievement motivation, Wiley and Sons, New York, 1966.

[17] J. W. Atkinson and W. Lens, Fähigkeit und Motivation als Determinanten momentaner und kumulativer Leistung, in: Fähigkeiten und Motivation in erwartungswidriger Schulleistung, (H. Heckhausen, Hg.), Hogrefe, Göttingen, 1980, 129-192. 


$$
\text { "herber" — 2006/6/26 — 10:27 — page } 40 \text { — \#40 }
$$

[18] J. W. Atkinson, Motivational determinants of risk-taking behavior, Psychological Review 64 (1957), 359-372.

[19] J. W. Atkinson, Strength of motivation and efficiency of performance, in: Motivation and achievement, (J. W. Atkinson and J. O. Raynor, eds.), Winston, Washington, 1974a, 193-218.

[20] J. W. Atkinson, Motivational determinants of intellective performance and cumulative achievement, in: Motivation and achievement, (J. W. Atkinson and J. O. Raynor, eds.), Winston, Washington, 1974b, 389-410.

[21] J. W. Atkinson, Motivational determinants of thematic apperception, in: Motivation and personality: Handbook of thematic content analysis, (C. P. Smith, ed.), University Press, Cambridge, 1992, 21-48.

[22] A. Bandura, Lernen am Modell. Ansätze zu einer sozial-kognitiven Lerntheorie, Klett, Stuttgart, 1976.

[23] A. Bandura, Self-efficacy: The exercise of control, Freeman, New York, 1997.

[24] D. E. Berlyne, Conflict, arousal, and curiosity, McGraw-Hill, New York, 1960.

[25] D. E. Berlyne, Curiosity and learning, Motivation and Emotion 2 (1978), 97-175.

[26] K. C. Berridge and P. Winkielman, What is an unconscious emotion?, Cognition and Emotion 17 (2003), 181-211.

[27] M. Boekaerts and P. Boscolo, Interest in learning, learning to be interested, Learning and Instruction 12 (2002), 375-382.

[28] V. Brandstätter, Von der Schwierigkeit, Ziele aufzugeben: Wenn sich das Streben nach Erfreulichem in Vermeiden von Unerfreulichem verkehrt, in: Ziele und Wille in der Psychologie: Grundlagen und Anwendungen, (O. L. Braun, Hg.), Verlag empirische Pädagogik, Landau, 1988, 51-68.

[29] J. W. Brehm, A theory of psychological reactance, Academic Press, New York, 1966.

[30] T. W. Britt, Motivational and emotional consequences of self-engagement: Voting in the 2000 U.S. Presidential Election, Motivation and Emotion 27 (2003), 339-358.

[31] J. C. Brunstein, Implizite Motive und motivationale Selbstbilder, in: Diagnostik von Motivatin und Selbstkonzept, (J. Stiensmeier-Pelster und F. Rheinberg, Hg.), Hogrefe, Göttingen, 2003, 59-88.

[32] S.-A. Christianson (eds.), The handbook of emotion and memory, Erlbaum, Hillsdale, 1992.

[33] J. Cooper, P. A. Taves and M. P. Zanna, Arousal as a necessary condition for attitude change following induced compliance, Journal of Personality and Social Psychology 36 (1978), 1101-1106.

[34] F. Copei, Der fruchtbare Moment im Bildungsprozeß, Spektrum, Heidelberg, 1960.

[35] M. V. Covington, Making the grade. A self-worth perspective on motivation and school reform, University Press, Cambridge, 1992. 
[36] J. Crocker and L. E. Park, Seeking self esteem: Construction, maintainance, and protection of self-worth, in: Handbook of self and identity,, (M. R. Leary and J. P. Tangney, eds.), Guilford, New York, 2003, 291-313.

[37] A. R. Damasio, Der Spinoza-Effekt. Wie Gefühle unser Leben bestimme, List, München, 2003.

[38] R. DeCharms, Personal causation: The internal affective determinants of behavior, Academic Press, New York, 1968.

[39] R. DeCharms, Ein schulisches Trainingsprogramm zum Erleben eigener Verursachung, in: Bedingungen des Bildungsprozesses,, (W. Edelstein und D. Hopf, Hg.), Klett, Stuttgart, 1973, 60-78.

[40] E. L. Deci, Intrinsic motivation, Plenum, New York, 1975.

[41] E. L. Deci, Self-determined motivation and educational achievement, Advances in motivation, (T. Gjesme and R. Nygard, eds.), Scandinavian University Press, Oslo, 1996, 195-209.

[42] E. L. Deci, R. Koestner and R. M. Ryan, Extrinsic rewards and intrinsic motivation in education: Reconsidered again, Review of Educational Research 71 (2001), 1-27.

[43] E. L. Deci, N. Spiegel, R. Ryan, R. Koestner and M. Kauffman, Effects of performance standards of teaching styles: Behavior of controlling teachers, Journal of Educational Psychology 74 (1982), 852-859.

[44] E. M. Deci and R. M. Ryan, The "what" and „why“ of goal pursuits: Human needs and the self-determination of behavior, Psychological Inquiry 11 (2000), 227-268.

[45] O. Dickhäuser und F. Rheinberg, Bezugsnormorientierung: Erfassung, Probleme, Perspektiven, in: Diagnostik von Motivatin und Selbstkonzept,, (J. StiensmeierPelster und F. Rheinberg, Hg.), Hogrefe, Göttingen, 2003, 41-55.

[46] K. Dieussaert, W. Schaeken, W. De Neys and G. d'Ydewalle, Initial belief state as predictor of belief revision, Current Psychology of Cognition 19 (2000), 277-288.

[47] K. Dieussaert, W. Schaeken and G. d'Ydewalle, A study of the belief revision process: The value of context and contra-evidence, in: Non-monotonic and uncertain reasoning in the focus of competing paradigms of cognition, Pre-proceedings of the 1st Salzburg Workshop on Paradigms of Cognition, University of Salzburg (Special Research Programme), 2002.

[48] C. Dippl, Das Phänomen Selbstwertgefühl - Teile - Prozesse - Wesensmerkmale - in den Übergängen des Prozesses der Leistungsmotivation, Unveröff., Salzburg, 2004, Dissertation.

[49] C. Doering, Kohärenzgefühl: Grundlage für Selbstwertgefühl, Gesundheit und Leistung von Schulkindern, Pabst, Lengerich, 2004.

[50] D. Dörner, Bauplan für eine Seele,, Rowohlt, Reinbek, 1999.

[51] M. Dresel, Motivationsförderung im schulischen Kontext, Hogrefe, Göttingen, 2004.

[52] G. M. Edelman und G. Tononi, Gehirn und Geist, Beck, München, 2002.

[53] R. Elio and F. Pelletier, Belief change as propositional update, Cognitive Science 21 (1997), 419-460. 


$$
\text { "herber" — 2006/6/26 — 10:27 — page } 42 \text { — \#42 }
$$

[54] L. Eliot, Was geht da drinnen vor? Die Gehirnentwicklung in den ersten fünf Lebensjahren, Berlin Verlag, Berlin, 2001.

[55] N. Entwistle, V. McCune and P. Walker, Conceptions, styles, and approaches within higher education: Analytic abstractions and everyday experience, in: Perspectives on thinking, learning, and cognitive styles, (R. J. Sternberg and L. Zhang, eds.), Erlbaum, Mahwah, 2001, 103-136.

[56] M. W. Eysenck, Attention and arousal, Springer, Berlin, 1982.

[57] T. Farahat and H. Astleitner, The effectiveness of web-based instruction in educating teachers - a quasi-experimental field-study, Salzburger Beiträge zur Erziehungswissenschaft 8, Heft 1 (2004), 53-60.

[58] L. Festinger, A theory of cognitive dissonance, Row and Petersen, Evanston, 1957.

[59] N. A. Fox and S. D. Calkins, The development of self-control of emotion: Intrinsic and extrinsic influences, Motivation and Emotion 27 (2003), 7-26.

[60] S. Freud, Drei Abhandlungen zur Sexualtheorie, Fischer, Frankfurt, 1904-5/1965.

[61] S. Freud, Enwurf einer Psychologie, in: Aus den Anfängen der Psychoanalyse, (S. Freud), Fischer, Frankfurt, 1895/1962, 299-384.

[62] S. M. Fröhlich und J. Kuhl, Das Selbststeuerungsinventar: Dekomponierung volitionaler Funktionen, in: Diagnostik von Motivatin und Selbstkonzept, (J. Stiensmeier-Pelster und F. Rheinberg, Hg.), Hogrefe, Göttingen, 2003, 221-257.

[63] K. J. Fuchs, H.-J. Herber und É. Vásárhelyi, Ähnlichkeit - Analogie - Innere Differenzierung. Ein Bericht über ein Unterrichtsprojekt zum Thema Ähnlichkeit. Beiträge zum Mathematikunterricht, Franzbecker, Hildesheim, 2001, 205-208.

[64] M. Gagné, The role of autonomy support and autonomy orientation in prosocial behavior engagement, Motivation and Emotion 27 (2003), 199-223.

[65] W.-D. Gasztner, Innere Differenzierung und Individualisierung im Mathematikunterricht, in: Pädagogische Intentionen, (R. Vierlinger, Hg.), OÖ. Landesverlag, Linz, 1978, 37-49.

[66] E. Görlitz, Exploration and attribution in a developmental context, in: Curiosity, imagination, and play, (D. Görlitz and J. F. Wohlwill, eds.), Erlbaum, Hillsdale, 1987, 78-103.

[67] S. A. Greenfield, Reiseführer Gehirn, Spektrum Akademischer Verlag, Heidelberg, 1999.

[68] J.-E. Gustafsson and J. O. Undheim, Individual differences in cognitive functions, in: Handbook of educational psychology, (D. C. Berliner and R. C. Calfee, eds.), Macmillan Library, New York, 1996, 186-241.

[69] H. Halvari, The effect of motivation on goal orientation, energy release, and performance, in: Advances in motivation, (T. Gjesme and R. Nygard, eds.), Scandinavian University Press, Oslo, 1996, 178-194.

[70] S. Harter, The relationship between perceived competence, affect, and motivational orientation within the classroom: Process and patterns of change, in: Achievement and motivation: A social-development perspective, (A. Boggiano and T. Pittman, eds.), Cambridge University Press, New York, 1992, 77-114. 


$$
\text { "herber" — 2006/6/26 — 10:27 — page } 43 \text { — \#43 }
$$

Kompetenzstreben und Kompetenzerwerb: Funktionale didaktische Fördermöglichkeiten. . 43

[71] H. Heckhausen, Hoffnung und Furcht in der Leistungsmotivation, Hain, Meisenheim, 1963.

[72] H. Heckhausen, Leistung und Chancengleichheit, Hogrefe, Göttingen, 1974.

[73] H. Heckhausen, Achievement motivation and its constructs: A cognitive model, Motivation and Emotion 1 (1977), 283-329.

[74] H. Heckhausen, Motivation und Handeln, Springer, Berlin, 1989.

[75] F. Heider, The psychology of interpersonal relations, Wiley, New York, 1958, (deutsch: Psychologie der interpersonalen Beziehungen, Klett, Stuttgart, 1977).

[76] A. Helmke, Selbstvertrauen und schulische Leistungen, Hogrefe, Göttingen, 1992.

[77] A. Helmke, Motivation und Schulleistung: Trends, Sackgassen und Perspektiven der Forschung, in: Bericht über den 38. Kongreß der Deutschen Gesellschaft für Psychologie in Trier 1992. Bd. 2, (L. Montada, ed.), Hogrefe, Göttingen, 1993, 588-595.

[78] H.-J. Herber, Die Bedeutung der Leistungsmotivation für die Selbstverwirklichung, Unveröff., Salzburg, 1972, Dissertation.

[79] H.-J. Herber, Motivationspsychologie, Kohlhammer, Stuttgart, 1976.

[80] H.-J. Herber, Das Verhältnis von intrinsischer und extrinsischer Motivation. Oder: Was das Lernen eigentlich lernenswert macht, Erziehung und Unterricht 127 (1977), 559-568.

[81] H.-J. Herber, Motivationstheorie und pädagogische Praxis, Kohlhammer, Stuttgart, 1979.

[82] H.-J. Herber, Innere Differenzierung im Unterricht, Kohlhammer, Stuttgart, 1983.

[83] H.-J. Herber, Das Theorie-Praxis-Problem, in: Die Guten ins Töpfchen, die Schlechten ...? (R. Vierlinger, Hg.), Haller, Passau, 1987, 43-57.

[84] H.-J. Herber, Innere Differenzierung, Unser Weg 49 (1994), 121-131.

[85] H.-J. Herber, Motivationsanalyse, Expert, Renningen-Malmsheim, Linde, Wien, 1998a.

[86] H.-J. Herber, Ein Paradigmenvergleich: „Interesse“ aus pädagogischer und psychologischer Sicht, in: Koexistenz rivalisierender Paradigmen, (G. Schurz und P. Weingartner, Hg.), Westdeutscher Verlag, Opladen, 1998b, 141-151.

[87] H.-J. Herber, Das Unterrichtsmodell „Innere Differenzierung“. Die Bedeutung von Analogiebildungs- und Motivationsprozessen, in: Bildungswege, (W. Lenz, Hg.), STUDIENVerlag, Innsbruck, 1998c, 59-71.

[88] H.-J. Herber, Innere Differenzierung, Pädagogisches Handeln 2 (1998d), 69-82.

[89] H.-J. Herber, Von der Selbstermöglichung zur Selbstverwirklichung, in: Erziehung - Therapie - Sinn, (A. A. Bucher, Hg.), LIT, Münster, 2004, 107-125.

[90] H.-J. Herber, R. Kainz und R. Vierlinger, Eine Variation zum Thema Gesamtschule, in: Pädagogische Intentionen, (R. Vierlinger, Hg.), OÖ. Landesverlag, Linz, 1978, 11-35. 


$$
\text { "herber" — 2006/6/26 — 10:27 — page } 44 \text { — \#44 }
$$

[91] H.-J. Herber, H. Astleitner und E. Faulhammer, Musikunterricht und Leistungsmotivation, Salzburger Beiträge zur Erziehungswissenschaft 3, Heft 2 (1999), 41-67.

[92] H.-J. Herber, H. Astleitner, É. Vásárhelyi und K. J. Parisot, Paradigmen des Lehrens und Lernens. Theoretische Überlegungen um ein Feldexperiment, in: Grundrisse einer humanen Schule, (S. Popp, Hg.), STUDIENVerlag, Innsbruck, 1998e, 92-120.

[93] H.-J. Herber, H. Astleitner, É. Vásárhelyi und K. J. Parisot, Analogisierende versus sequentielle Instruktion, experimentell induzierte Aufgabenschwierigkeiten und Mathematikleistungen, in: Situationsspezifität in pädagogischen Handlungsfeldern, (J.-L. Patry und F. Riffert, Hg.), STUDIENVerlag, Innsbruck, 2000, 125-136.

[94] H.-J. Herber, E. Faulhammer und A. Paschon, Musikunterricht und Leistungsmotivation, Teil II, Salzburger Beiträge zur Erziehungswissenschaft 5, Heft 2 (2001), $5-26$.

[95] H.-J. Herber und É. Vásárhelyi, Lewins Feldtheorie als Hintergrundsparadigma moderner Motivations- und Willensforschung (im Vergleich zu Behaviorismus, Psychoanalyse, Gestalt- und Kognitionspsychologie), Salzburger Beiträge zur Erziehungswissenschaft 6, Heft 1 (2002a), 37-100.

[96] H.-J. Herber und É. Vásárhelyi, Das Unterrichtsmodell „Innere Differenzierung einschließlich Analogiebildung“, Salzburger Beiträge zur Erziehungswissenschaft 6, Heft 2 (2002b), 5-19.

[97] H.-J. Herber und É. Vásárhelyi, Moderne Motivationsforschung als Paradigmenverschmelzung?, Salzburger Beiträge zur Erziehungswissenschaft 7, Heft 2 (2003), $5-60$.

[98] H.-J. Herber und É. Vásárhelyi, Kompetenzstreben und Kompetenzerwerb: Funktionale Dekomponierung und didaktische Fördermöglichkeiten, Salzburger Beiträge zur Erziehungswissenschaft 8, Heft 2 (2004), 5-34.

[99] S. Hidi, Interest and its contribution as a mental resource for learning, Review of Educational Research 60 (1990), 549-571.

[100] S. Hidi, D. Berndorff and M. Ainley, Children's argument writing, interest and self-efficacy: an intervention study, Learning and Instruction 12 (2002), 429-446.

[101] L. Hoffmann, Promoting girls' interest and achievement in physics classes for beginners, Learning and Instruction 12 (2002), 447-465.

[102] R. M. Hogarth and H. J. Einhorn, Order effects in belief updating: The belief-adjustment model, Cognitive Psychology 24 (1992), 1-55.

[103] M. S. Horner, The measurement and behavioral implications of fear of success in women, in: Motivation and achievement, (J. W. Atkinson and J. O. Raynor, eds.), Winston, Washington, 1974a, 91-117.

[104] M. S. Horner, Performance of men in noncompetitive and interpersonal competitive achievement-oriented situations, in: Motivation and achievement, (J. W. Atkinson and J. O. Raynor, eds.), Winston, Washington, 1974b, 237-254.

[105] G. L. Huber, Processes of decision-making in small learning groups, Learning and instruction 13 (2003), 255-269. 


$$
\text { "herber" — 2006/6/26 — 10:27 — page } 45 \text { — \#45 }
$$

Kompetenzstreben und Kompetenzerwerb: Funktionale didaktische Fördermöglichkeiten. . 45

[106] C. L. Hull, Principles of behavior, Appleton-Century-Crofts, New York, 1943.

[107] S. A. Karabenick and Z. I. Youssef, Performance as a function of achievement motive level and perceived difficulty, in: Motivation and achievement, (J. W. Atkinson and J. O. Raynor, eds.), Winston, Washington, 1974, 83-90.

[108] T. Kasser and R. M. Ryan, Be careful what you wish for: Optimal functioning and the relative attainment of intrinsic and extrinsic goals, in: Life goals and well-being, (P. Schmuck and K. M. Sheldon, eds.), Hogrefe and Huber, Seattle, 2001, 116-131.

[109] M. Kazén, N. Baumann and J. Kuhl, Self-infiltration vs. self-compatibility checking in dealing with unattractive tasks: The moderating influence of state vs. action orientation, Motivation and Emotion 27 (2003), 157-197.

[110] G. A. Kimble, Evolution of the nature-nurture issue in the history of psychology, in: Nature, nurture, and psychology, (R. Plomin and G. E. McClearn, eds.), American Psychological Association, Washington, 1993.

[111] D. Klahr, P. Langley and R. Neches, Production system models of learning and development, The MIT Press, Cambridge, 1987.

[112] F. Klix, Die Natur des Verstandes, Hogrefe, Göttingen, 1992.

[113] F. Klix, Erwachendes Denken. Geistige Leistungen aus evolutionspsychologischer Sicht, Spektrum Akademischer Verlag, Heidelberg, 1993.

[114] H. Kohut, The restoration of the self, International University Press, New York, 1977, (deutsch: Die Heilung des Selbst, Suhrkamp, Frankfurt, 1979).

[115] A. Krapp und M. Prenzel (Hg.), Interesse, Lernen, Leistung, Aschendorff, Münster, 1992.

[116] A. Krapp, Structural and dynamic aspects of interest development: theoretical considerations from an ontogenetic perspective, Learning and Instruction 12 (2002), 383-409.

[117] J. Kuhl und M. Kazén, Handlungs- und Lageorientierung: Wie lernt man, seine Gefühle zu steuern?, Diagnostik von Motivation und Selbstkonzept, (F. Rheinberg und J. Stiensmeier-Pelster, Hg.), Hogrefe, Göttingen, 2003, 201-219.

[118] J. Kuhl, Motivational chaos: A simple model, in: Frontiers of motivational psychology, Essays in honor of John W. Atkinson, (D. R. Brown and J. Veroff, eds.), Springer, Berlin, 1986, 54-71.

[119] J. Kuhl, Motivation und Persönlichkeit, Interaktionen psychischer Systeme, Hogrefe, Göttingen, 2001.

[120] J. Kuhl, D. Scheffer und J. Eichstaedt, Der Operante Motiv-Test (OMT): Ein neuer Ansatz zur Messung impliziter Motive, in: Diagnostik von Motivation und Selbstkonzept, (F. Rheinberg und J. Stiensmeier-Pelster, Hg.), Hogrefe, Göttingen, 2003, 129-149.

[121] T. A. Langens und J. Schüler, Die Messung des Leistungsmotivs mittels des Thematischen Auffassungstests, in: Diagnostik von Motivation und Selbstkonzept, (F. Rheinberg und J. Stiensmeier-Pelster, Hg.), Hogrefe, Göttingen, 2003, 89-104.

[122] M. R. Leary and J. P. Tangney (eds.), Handbook of self and identity, Guilford, New York, 2003. 


$$
\text { "herber" — 2006/6/26 — 10:27 — page } 46 \text { — \#46 }
$$

[123] R. Lehrer, Computer-assisted strategic instruction, in: Cognitive strategy research: From basic research to educational applications, (C. B. McCormick, G. Miller and M. Pressley, eds.), Springer, New York, 1989, 303-320.

[124] D. Leutner, A. Barthel und B. Schreiber, Studierende können lernen, sich selbst zum Lernen zu motivieren: Ein Trainingsexperiment, Zeitschrift für Pädagogische Psychologie 15 (2001), 155-167.

[125] D. Lewalter und I. Schreyer, Entwicklung von Interessen und Abneigungen - zwei Seiten einer Medaille?, in: Interesse und Lernmotivation: Untersuchungen zu Entwicklung, Förderung und Wirkung, (U. Schiefele und K.-P. Wild, Hg.), Waxmann, Berlin, 2000, 53-72.

[126] D. Lewalter, Lernen mit Bildern und Animationen, Waxmann, Münster, 1997.

[127] D. Lewalter, Emotionales Erleben und Lernmotivation - Theoretische und empirische Analyse des Zusammenhangs von Emotion und Motivation in pädagogischen Kontexten, Unveröff., München, 2002, Habilitationsschrift.

[128] K. Lewin, Vorsatz, Wille und Bedürfnis, Springer, Berlin, 1926.

[129] K. Lewin, Feldtheorie, in: Kurt-Lewin-Werkausgabe Bd. 4, (C.-F. Graumann, Hg.), Huber, Bern, Klett-Cotta, Stuttgart, 1982, 41-312.

[130] K. Lewin, T. Dembo, L. Festinger and P. S. Sears, Level of aspiration, in: Personality and the behavior disorders, Vol. 1, (J. M. Hunt, ed.), Ronald Press, New York, 1944, 333-344.

[131] H. Lukesch, Lehren unter Berücksichtigung von Metakognition und Visualisierung am Beispiel der Technik der Begriffslandschaften („concept maps“), in: Grundrisse einer humanen Schule, (S. Popp, Hg.), STUDIENVerlag, Innsbruck, 1998, $121-141$.

[132] H. Lukesch,, Psychologie des Lernens und Lehrens, Roderer, Regensburg, 2001.

[133] C. J. Lumsden and A. C. Gushurst, Gene-culture coevolution: Humankind in the making, in: Sociobiology and epistemology, (J. H. Fetzer, ed.), Reidel, Dordrecht, 1985.

[134] M. L. Maehr and P. R. Pintrich (eds.), Advances in motivation and achievement, Vol. 7, Jai, Greenwich, 1991.

[135] A. H. Maslow, A theory of human motivation, Psychological Review 50 (1943), 370-396.

[136] A. H. Maslow, Motivation and personality, Harper and Row, New York, 1954, (deutsch: Motivation und Persönlichkeit, Walter Verlag, Olten, 1977).

[137] D. C. McClelland and R. Koestner, The achievement motive, in: Motivation and personality: Handbook of thematic content analysis, (C. P. Smith, ed.), University Press, Cambridge, 1992, 143-152.

[138] D. C. McClelland and G. Teague, Predicting risk preferences among power related tasks, Journal of Personality 43 (1975), 266-285.

[139] D. C. McClelland, Motives, personality, and society, Praeger, New York, 1984.

[140] D. C. McClelland and R. I. Watson, Power motivation and risk-taking behavior, Journal of Personality 41 (1973), 121-138. 


$$
\text { "herber" — 2006/6/26 — 10:27 — page } 47 \text { — \#47 }
$$

Kompetenzstreben und Kompetenzerwerb: Funktionale didaktische Fördermöglichkeiten. . 47

[141] D. C. McClelland, Human motivation, University Press, Cambridge, 1987.

[142] D. C. McClelland, J. W. Atkinson, R. A. Clark and E. L. Lowell, The achievement motive, Appleton-Century-Crofts, New York, 1953.

[143] D. C. McClelland, R. Koestner and J. Weinberger, How do self-attributed and implicit motives differ?, in: Motivation and personality: Handbook of thematic content analysis, (C. P. Smith, ed.), University Press, Cambridge, 1992, 49-72.

[144] D. B. McLeod and V. M. Adams, Affect and mathematical problem solving, Springer, London, 1989.

[145] D. McMullin and J. Steffen, Intrinsic motivation and performance standards, Social Behavior and Personality 10 (1982), 47-56.

[146] W.-U. Meyer, Gelernte Hilflosigkeit: Grundlagen und Anwendungen in Schule und Unterricht, Huber, Bern, 2000.

[147] R. W. Moulton, Motivational implications of individual differences in competence, in: Motivation and achievement, (J. W. Atkinson and J. O. Raynor, eds.), Winston, Washington, 1974, 77-82.

[148] H. Neber (Hg.), Entdeckendes Lernen, Beltz, Weinheim, 1981.

[149] H. Neber (Hg.), Angewandte Problemlösepsychologie, Aschendorff, Münster, 1987.

[150] P. Nenniger, Das Pädagogische Verhältnis als motivationales Konstrukt, Deutscher Studien Verlag, Weinheim, 1988.

[151] P. Nenniger, G. Eigler and G. Macke, Studien zur Mehrdimensionalität von LehrLern-Prozessen, Lang, Bern, 1993.

[152] J. G. Nicholls, M. Patashnick, P. C. Cheung, T. A. Thorkildsen and J. M. Lauer, Can achievement theory succeed with only one conception of success?, in: International perspectives on achievement and task motivation, (F. Halisch and J. van den Bercken, eds.), Swets and Zeitlinger, Amsterdam, 185-208.

[153] C. Peacocke, Being known, Clarendon Press, Oxford, 1999.

[154] J. Piaget, Psychologie der Intelligenz, Klett-Cotta, Stuttgart, 1980.

[155] P. Pintrich and E. De Groot, Motivational and self-regulated learning components of classroom academic performance, Journal of Educational Psychology 82 (1990), 33-40.

[156] G. Politzer and L. Carles, Belief revision and uncertain reasoning, Thinking and Reasoning 7 (2001), 217-234.

[157] E. Pöppel, Grenzen des Bewußtseins, Insel, Frankfurt, 2000.

[158] B. Priemer und L.-H. Schön, Textproduktionsverfahren von Schülern beim Lernen mit dem Internet, Psychologie in Erziehung und Unterricht 51 (2004), 89-98.

[159] T. Pyszczynski, J. Greenberg and J. L. Goldenberg, Freedom versus fear: On the defense, growth, and expansion of the self, in: Handbook of self and identity, (M. R. Leary and J. P. Tangney, eds.), Guilford, New York, 2003, 314-343.

[160] J. O. Raynor and I. S. Rubin, Effects of achievement motivation and future orientation on level of performance, in: Motivation and achievement, (J. W. Atkinson and J. O. Raynor, eds.), Winston, Washington, 1974, 181-191. 


$$
\text { "herber" — 2006/6/26 — 10:27 — page } 48 \text { — \#48 }
$$

[161] J. O. Raynor, Relationships between achievement-related motives, future orientation, and academic performance, in: Motivation and achievement, (J. W. Atkinson and J. O. Raynor, eds.), Winston, Washington, 1974, 173-180.

[162] K. A. Renninger, L. Ewen and A. K. Lasher, Individual interest as context in expository text and mathematical word problems, Learning and Instruction 12 (2002), 467-491.

[163] J. S. Renzulli and D. Y. Dai, Abilities, interests, and styles as aptitudes for learning: A person-situation interaction perspective, in: Perspectives on thinking, learning, and cognitive styles, (R. J. Sternberg and L. Zhang, eds.), Erlbaum, Mahwah, 2001, 23-46.

[164] F. Rheinberg, Leistungsbewertung und Lernmotivation, Hogrefe, Göttingen, 1980.

[165] F. Rheinberg, Motivation, Kohlhammer, Stuttgart, 2002.

[166] F. Rheinberg, Motivationsdiagnostik, Hogrefe, Göttingen, 2004.

[167] R. Riding, The nature and effects of cognitive style, in: Perspectives on thinking, learning, and cognitive styles, (R. J. Sternberg and L. Zhang, eds.), Erlbaum, Mahwah, 2001, 47-72.

[168] R. Riedl, Biologie der Erkenntnis, Parey, Hamburg, 1980.

[169] M. Riketta and D. Dauenheimer, Anticipated success at unconscious goal pursuit: Consequences for mood, self-esteem, and the evaluation of a goal-relevant task, Motivation and Emotion 27 (2003), 327-338.

[170] J. B. Rotter, Generalized expectancies for internal versus external control of reinforcement, Psychological Monographs: General and Applied 80 (1966), 1-28.

[171] J. B. Rotter, J. E. Chance and E. J. Phares (eds.), Applications of a social learning theory of personality, Holt, Rinehart and Winston, New York, 1972.

[172] L. Satow and R. Schwarzer, Perceived self-efficacy, worry, and school achievement. A longitudinal study, Empirische Pädagogik 15 (2000), 177-214.

[173] W. Schaeken, G. De Vooght, A. Vandierendonck and G. d'Ydewalle, Deductive reasoning and strategies, Erlbaum, Mahwah, 2000.

[174] D. Scheffer, Messung von Motiven, Hogrefe, Göttingen, 2003.

[175] D. Scheffer, J. Kuhl und J. Eichstaedt, Der Operante Motiv-Test (OMT): Inhaltsklassen, Auswertung, psychometrische Kennwerte und Validierung, in: Diagnostik von Motivation und Selbstkonzept, (F. Rheinberg und J. Stiensmeier-Pelster, Hg.), Hogrefe, Göttingen, 2003, 151-168.

[176] R. Schiefele, Der Einfluß von Interesse auf Umfang, Inhalt und Struktur studienbezogenen Wissens, Zeitschrift f. Entwicklungspsychologie u. Pädagogische Psychologie 20 (1988), 356-370.

[177] U. Schiefele, Interest, learning, and motivation, Educational Psychologist 26 (1991), 299-323.

[178] U. Schimmack and R. Reisenzein, Experiencing activation: Energetic arousal and tense arousal are not mixtures of valence and activation, Emotion 2 (2002), $412-417$.

[179] K. Schleider, Komponenten der Gedächtnisentwicklung, Lang, Frankfurt, 1993. 


$$
\text { "herber" — 2006/6/26 — 10:27 — page } 49 \text { — \#49 }
$$

Kompetenzstreben und Kompetenzerwerb: Funktionale didaktische Fördermöglichkeiten. . 49

[180] C. Schleiss, „Buama stehts z'samm im Kroas ..." Das Lernen und Aufführen von Volkstänzen im Salzkammergut - im besonderen der in diesen enthaltenen geräuschimmanenten rhythmischen Handlungen, Unveröff., Salzburg, 2004, Diplomarbeit.

[181] H.-D. Schmalt, Leistungsmotivation im Unterricht: über den Einsatz des LM-Gitters in der Schule, in: Diagnostik von Motivation und Selbstkonzept, (J. Stiensmeier-Pelster und F. Rheinberg, Hg.), Hogrefe, Göttingen, 2003, 105-127.

[182] G. S. Schmitz und R. Schwarzer, Selbstwirksamkeitserwartung von Lehrern: Längsschnittbefunde mit einem neuen Instrument, Zeitschrift für Pädagogische Psychologie 14 (2000), 12-25.

[183] P. Schmuck and K. M. Sheldon (eds.), Life goals and well-being, Hogrefe and Huber, Seattle, 2001.

[184] K. J. Schneider, The paradoxical self, Plenum Press, New York, 1990.

[185] W. Schneider and F. E. Weinert, The role of knowledge, strategies, and aptitudes in cognitive performance: Concluding comments, in: Interactions among aptitudes, strategies, and knowledge in cognitive performance, (W. Schneider and F. E. Weinert, eds.), Springer, New York, 1990.

[186] C. Schöne, O. Dickhäuser, B. Spinath und J. Stiensmeier-Pelster, Das Fähigkeitsselbstkonzept und seine Erfassung, in: Diagnostik von Motivation und Selbstkonzept, (J. Stiensmeier-Pelster und F. Rheinberg, Hg.), Hogrefe, Göttingen, 2003, 3-14.

[187] G. Schurz, Was ist „normal“? Normische Gesetze und ihre evolutionstheoretische Begründung, SFB F012 Forschungsmitteilungen, Salzburg, 2001, Ausgabe 15.

[188] P. A. Schutz, Goals as the transactive point between motivation and cognition, in: Student motivation, cognition, and learning, (P. R. Pintrich, D. R. Brown and C. E. Weinstein, eds.), Erlbaum, Hillsdale, 1994, 135-156.

[189] N. M. Seel, Psychologie des Lernens, Reinhardt, München, 2000.

[190] J. A. Seidler, Information systems and data compression, Kluwer, Boston, 1997.

[191] M. E. P. Seligman, Helplessnesss: On depression, development, and death, Freeman, San Francisco, 1975, (deutsch: Erlernte Hilflosigkeit, Urban and Schwarzenberg, München, 1983).

[192] M. P. Shaw and M. A. Runco (eds.), Creativity and affect, Ablex, Norwood, 1994.

[193] K. M. Sheldon, The self-concordance model of healthy goal striving: When personal goals correctly represent the person, in: Life goals and well-being, (P. Schmuck and K. M. Sheldon, eds.), Hogrefe and Huber, Seattle, 2001, 18-36.

[194] W. Singer, Der Beobachter im Gehirn, Suhrkamp, Frankfurt, 2002.

[195] M. Slusarek, Neugier und Problemlösen, Waxmann, Münster, 1995.

[196] R. E. Snow, L. Corno and D. Jackson, Individual differences in affective and conative functions, in: Handbook of educational psychology, (D. C. Berliner and R. C. Calfee, eds.), Macmillan Library, New York, 1996, 243-310.

[197] K. Spies und G. Lüer, Intelligenz als Fähigkeit zum Problemlösen, in: Intelligenz, (E. Roth, Hg.), Kohlhammer, Stuttgart, 1998, 185-215. 


$$
\text { "herber" — 2006/6/26 — 10:27 — page } 50 \text { — \#50 }
$$

[198] K. Spies, Negative Stimmung und kognitive Verarbeitungskapazität, Waxmann, Münster, 1995.

[199] B. Spinath und C. Schöne, Subjektive Überzeugungen zu Bedingungen von Erfolg in Lern- und Leistungskontexten und deren Erfassung, in: Diagnostik von Motivation und Selbstkonzept, (J. Stiensmeier-Pelster und F. Rheinberg, Hg.), Hogrefe, Göttingen, 2003, 15-27.

[200] J. Standop, Emotionen und kognitives schulisches Lernen aus interdisziplinärer Perspektive. Emotions-psychologische, neuropsychologische und schulpädagogische Zusammenhänge - ihre Berücksichtigung im schulischen Bildungsauftrag wie den Forschungen zum Unterrichtsklima und der Klassenführung, Lang, Frankfurt, 2002.

[201] R. J. Sternberg and L. Zhang (eds.), Perspectives on thinking, learning, and cognitive styles, Erlbaum, Mahwah, 2001.

[202] J. Stiensmeier-Pelster und F. Rheinberg (Hg.), Diagnostik von Motivation und Selbstkonzept, Hogrefe, Göttingen.

[203] D. J. Stipek, Motivation and instruction, in: Handbook of educational psychology, (D. C. Berliner and R. C. Calfee, eds.), Macmillan Library, New York, 1996, 85-113.

[204] D. J. Stipek, Motivation to learn, Allyn and Bacon, Boston, 1998.

[205] J. Strelau and H. J. Eysenck, Personality dimensions and arousal, Plenum Press, New York, 1987.

[206] H.-M. Süß, Intelligenz, Wissen und Problemlösen, Hogrefe, Göttingen, 1996.

[207] R. E. Thayer, Toward a psychological theory of multidimensional activation (arousal), Motivation and Emotion 1 (1978), 1-34.

[208] R. E. Thayer, The biopsychology of mood and arousal, Oxford University Press, New York, 1989.

[209] B. Torff and R. J. Sternberg, Understanding and teaching the intuitive mind, Erlbaum, Mahwah, 2001.

[210] E. Van der Meer, Intelligenz als Informationsverarbeitung, in: Intelligenz, (E. Roth, Hg.), Kohlhammer, Stuttgart, 1998, 161-184.

[211] B. Van Oers, The dynamics of school learning, in: The structure of learning processes, (J. Valsiner and H.-G. Voss, eds.), Ablex, Norwood, 1996, 205-228.

[212] M. Vansteenkiste and E. L. Deci, Competitively contingent rewards and intrinsic motivation: Can losers remain motivated?, Motivation and Emotion 27 (2003), 273-299.

[213] É. Vásárhelyi, Analogie und Problemlösen, in: Trends im Geometrieunterricht, (K. J. Parisot und É. Vásárhelyi, Hg.), Abakus, Salzburg, 1996, 87-94.

[214] É. Vásárhelyi, Combination of traditional and computer based tools as a strategy for problem solving, in: Creativity and mathematics education, (University Münster, ed.), Tagungsband, Münster, 1999, 163-166. 


$$
\text { "herber" — 2006/6/26 — 10:27 — page 51 — \#51 }
$$

Kompetenzstreben und Kompetenzerwerb: Funktionale didaktische Fördermöglichkeiten. . 51

[215] É. Vásárhelyi, Experimentieren um einen Satz zu finden - vollständig separierbare Mosaike auf der Kugel und ihre Anwendungen, Teaching Mathematics and Computer Science 1, no. 2, Debrecen (2003), 297-319.

[216] É. Vásárhelyi, Aufgaben und Lösungen im Sinne der Inneren Differenzierung, Salzburger Beiträge zur Erziehungswissenschaft 8, Heft 1 (2004a), 61-76.

[217] É. Vásárhelyi, Bemerkungen zur Prototypentheorie, Forschungsbericht, Salzburg, 2004b, Mathematikdidaktisches Forum: http://www.mathdid.inhun.com.

[218] É. Vásárhelyi, Elektronische Arbeitsblätter, 2004c, Mathematikdidaktisches Forum: http://www. mathdid.inhun.com.

[219] É. Vásárhelyi, Problemlösen durch geometrische Repräsentation, 2004d, Mathematikdidaktisches Forum: http://www.mathdid.inhun.com.

[220] J. Wegge, Lernmotivation, Informationsverarbeitung, Leistung, Waxmann, Münster, 1998.

[221] B. Weiner, Theories of motivation. From mechanism to cognition, Rand McNally, Chicago, 1972.

[222] B. Weiner, The effects of unsatisfied achievement motivation on persistence and subsequent performance, in: Motivation and achievement, (J. W. Atkinson and J. O. Raynor, eds.), Winston, Washington, 1974a, 347-357.

[223] B. Weiner, Role of success and failure in the learning of easy and complex tasks, in: Motivation and achievement, (J. W. Atkinson and J. O. Raynor, eds.), Winston, Washington, 1974b, 359-368.

[224] B. Weiner, Human motivation. Metaphors, theories, and research, Sage, Newbury Park, 1996.

[225] B. Weiner, I. Frieze, A. Kukla, L. Reed, S. Rest and R. M. Rosenbaum, Perceiving the causes of success and failure, in: Attribution: Perceiving the causes of behavior, (E. E. Jones, E. E. Kanouse, H. H. Kelley, R. E. Nisbett, S. Valins and B. Weiner, eds.), General Learning Press, Morristown, 1971, 95-120.

[226] M. Wertheimer, Experimentelle Studien über das Sehen von Bewegung, Zeitschrift für Psychologie 61 (1912), 161-265.

[227] R. W. White, Motivation reconsidered: The concept of competence, Psychological Review 66 (1959), 297-333.

[228] H. Wiesner, Bildung als Vektor der Nachhaltigkeit, Unveröff., Salzburg, 2004, Dissertation.

[229] S. Wolfram, A new kind of science, Wolfram Media, Champaign, 2002.

[230] R. M. Yerkes and J. D. Dodson, The relation of strength of stimulus to rapidity of habit-formation, Journal of Comparative Neurology and Psychology 18 (1908), 459-482.

[231] F. Zech, Grundkurs Mathematikdidaktik, Beltz, Weinheim, 1998.

[232] B. J. Zimmerman and D. H. Schunk, Self-regulated learning and academic achievement, Springer, New York, 1989. 
HANS-JÖRG HERBER

UNIVERSITÄT SALZBURG FACHBEREICH FÜR ERZIEHUNGSWISSENSCHAFT UND

KULTURSOZIOLOGIE ABTEILUNG ERZIEHUNGSWISSENSCHAFT

A-5020 SALZBURG

AKADEMIESTRASSE 26

ÖSTERREICH

E-mail: hans-joerg.herber@sbg.ac.at

ÉVA VÁSÁRHELYI

EÖTVÖS LORÁND UNIVERSITÄT MATHEMATISCH-FACHDIDAKTISCHE GRUPPE

H-1117 BUDAPEST

PÁZMÁNY PÉTER SÉTÁNY 1/C

UNGARN

E-mail: vasar@ludens.elte.hu

(Received April, 2005) 